\title{
Diversity and distribution of cercosporoid fungi in Himachal Pradesh: an annotated checklist
}

\author{
Gautam AK ${ }^{1}$, Avasthi $\mathrm{S}^{2}$, Prasher IB $^{3}$, Sushma ${ }^{4}$, Verma RK ${ }^{5}$ \\ ${ }^{1}$ Faculty of Science, School of Agriculture, Abhilashi University, Mandi-175028, India \\ ${ }^{2}$ School of Studies in Botany, Jwaji University, Gwalior- 474011, India \\ ${ }^{3}$ Department of Botany, Mycology and Plant Pathology Laboratory, Panjab University Chandigarh -160014, India \\ ${ }^{4}$ Department of Agriculture, Dolphin PG College of science and Agriculture Chunni Kalan, Fatehgarh Sahib, Punjab, \\ 140307, India \\ ${ }^{5}$ Department of Agriculture, Swami Vivekanand Institute of Engineering and Technology, Banur, Punjab 140506, India
}

Gautam AK, Avasthi S, Prasher IB, Sushma, Verma RK 2020 - Diversity and distribution of cercosporoid fungi in Himachal Pradesh: an annotated checklist. Studies in Fungi 5(1), 17-49, Doi 10.5943/sif/5/1/3

\begin{abstract}
A checklist of cercosporoid fungi reported from Himachal Pradesh, India, has been prepared on the basis of available publications. A total of 103 species belonging to 15 genera of cercosporoid fungi have been reported from Himachal Pradesh on 118 plant species belonging to 46 families. The species richness of cercosporoid fungi in the region was as follows; Cercospora (50), Pseudocercospora (23), Ramularia and Passalora (7 each), Stigmina \& Mycosphaerella (3 each), Sirosporium (2), Cercosporella, Distomycovellosiella and Mycovellosiella, Neocercosporidium, Neopseudocercosporella, Nothopassalora, Rosisphaerella \& Teratosphaeria (1 each). The highest numbers of cercosporoid fungi were recorded on plant hosts of the family Solanaceae (12 species) followed by Fabaceae (10 species), Asteraceae and Rosaceae (8 species each), Amaranthaceae (5 species), Malvaceae and Smilaceae (4 species each), Acanthaceae, Poaceae, Polygonaceae and Ranunculaceae (3 species each), while the rest of families were found associated with 1-2 cercosporoid speices.
\end{abstract}

Key words - fungi - hyphomycetes - India - list - Mycosphaerellaceae - North Western Himalaya

\section{Introduction}

The term cercosporoid fungi refer to a large complex of fungi belonging to the Mycosphaerellaceae with a worldwide distribution. Most of them are plant pathogenic fungi that cause diseases on a wide range of hosts, including numerous cultivated plants. With a wide geographic distribution, these fungi are mostly foliar in nature associated with leaf spots on a broad range of plant hosts and are responsible for severe economic losses. However, in case of herbaceous plants, they can attack almost all aerial parts of the plants (Bakhshi et al. 2012, Braun et al. 2013).

The name of the group originates from Cercospora, a genus with continuously increasing group currently comprising more than 3000 species (Crous \& Braun 2003). Previously these fungi were named as mitosporic fungi or fungi imperfecti as mostly form asexual morphs and placed under anamorphic fungi of hyphomycetes. Some of them form mycosphaerella-like sexual morphs 
and positioned to the Mycosphaerellaceae (Ascomycota). A majority of anamorphic fungi are conidial stages of ascomycetous fungi (phylum: Ascomycota), and rarely basidiomycetous fungi (phylum: Basidiomycota) (Świderska-Burek 2015). Investigations of cercosporoid fungi have been conducted for over 150 years across the world and reported their wide host range. They are known to cause diseases on major agricultural crops (cereals, vegetables, ornamental plants, forest trees, etc.). They are also known to hyperparasites number of plant pathogens including rust fungi and can be evaluated as biocontrol agents in agriculture sector (Kamal 2010, Phengsintham et al. 2013, Świderska-Burek 2015, Braun et al. 2016, Bakhshi 2019).

Himachal Pradesh is a hilly state of northern India located within the latitudes $30^{\circ} 22^{\prime} 40^{\prime \prime}$ $33^{\circ} 12^{\prime} 40^{\prime \prime} \mathrm{N}$ and longitudes $75^{\circ} 44^{\prime} 55^{\prime \prime}-79^{\circ} 04^{\prime} 20^{\prime \prime} \mathrm{E}$. The state shows great variations in elevation ranging from about $350 \mathrm{~m}(1,148 \mathrm{ft})$ to 7,000 meters $(22,966 \mathrm{ft})$ above the sea level. It has very high mountains to plain grass lands with average rainfall in the range of 1500-3000 mm. Climatic variations of the state promote the abundance of rich biodiversity here. Total $66.52 \%$ of the area of the state is covered very dense evergreen to deciduous forests types. While, alpine shrub and meadows are found distributed in west and northeast Himalaya; alders, birches, rhododendrons and moist alpine shrubs are there as the regional vegetation. The plant pathogens including bacteria, fungi and viruses are also found these changeable geographical and climatic conditions of the state quite favourable for their growth and development. Additionally, these variable geo-climatic conditions of the state also promote overall growth of foliicolous fungi including cercosporoid fungi (Agrios 1997, Chakraborty et al. 1998, Coakley et al. 1999, Ahanger et al. 2013). Review of pertinent literature reveals that foliicolous fungi have been fairly well documented from some parts of India. Relevant literature on cercosporoid fungi of Himachal Pradesh is no doubt available but no thorough and collective inventory for the state. This compilation is prepared with an aim to understand the current status of distribution of cercosporoid fungi in the state of Himachal Pradesh.

\section{Materials \& Methods}

This checklist is prepared after an exhaustive bibliographic survey of the literature published on cercosporoid fungi of Himachal Pradesh in various national and international journals, monographs, books, book chapters and even magazines up to December 2019. The unpublished data and fungi identified up to genus level have not been included in this checklist. Some species names as reported in the cited publications have been replaced with their currently accepted name according to Index Fungorum (www.indexfungorum.org) as accessed on 06 December, 2019. The checklist arranged as fungal genera with brief taxonomical description, species names with their synonyms, host scientific names with family, literature cited. The host name given in the original citation is sometimes changed to be consistent with current taxonomy based on The Plant List (http://www.theplantlist.org). A geographical map of the state is provided in order to understand exact location of occurrence and distribution of the cercosporoid fungi in Himachal Pradesh (Fig. 1). The cases where species or a proper synonym was not recognized, species name cited originally is used. In order to understand the generic difference, line diagrams redrawn from original sourse with some modifications are also included in the paper.

\section{Results and Discussion}

A total 103 species of cercosporoid fungi belonging 15 genera have been identified and reported from Himachal Pradesh. The species richness in cercosporoid fungi in the region was as follows; Cercospora (50), Pseudocercospora (23), Ramularia (7), Passalora (7), Stigmina (3), Mycosphaerella (3), Sirosporium (2), Cercosporella, Distomycovellosiella, Mycovellosiella, Neocercosporidium, Neopseudocercosporella, Nothopassalora and Rosisphaerella \& Teratosphaeria (1each). Cercospora species were found associated with higest 59 host plants belonging to 50 genera and 25 families, including Solanaceae (9 species), Fabaceae (6 species), Asteraceae (5 species), Amaranthaceae (4 species), Acanthaceae \& Poaceae (3 species each) and Cucurbitaceae \& Malvaceae (2 species each). Similarly, Pseudocercospora species were reported to infect 25 host plants belonging to 23 genera and 21 families. The cercosporid fungi in the genus 
Ramularia were reported on 9 host plants of 8 genera of 8 familes while, Passalora on 7 host plants of 7 genera of 6 familes. Likewise, species of Stigmina were recorded on 3 host plants belonging to 3 genera and 3 families followed by Mycosphaerella (4 plant hosts of 3 genera and 3 families) and Sirosporium ( 2 plant hosts of 2 genera and 2 families). Rest of the cercosporoid fungi were reported on single plant host with single genus and family. The detailed information of all fungal genera and species along with thier host plants is presented in the list of cercosporoid fungi reported from Himachal Pradesh.

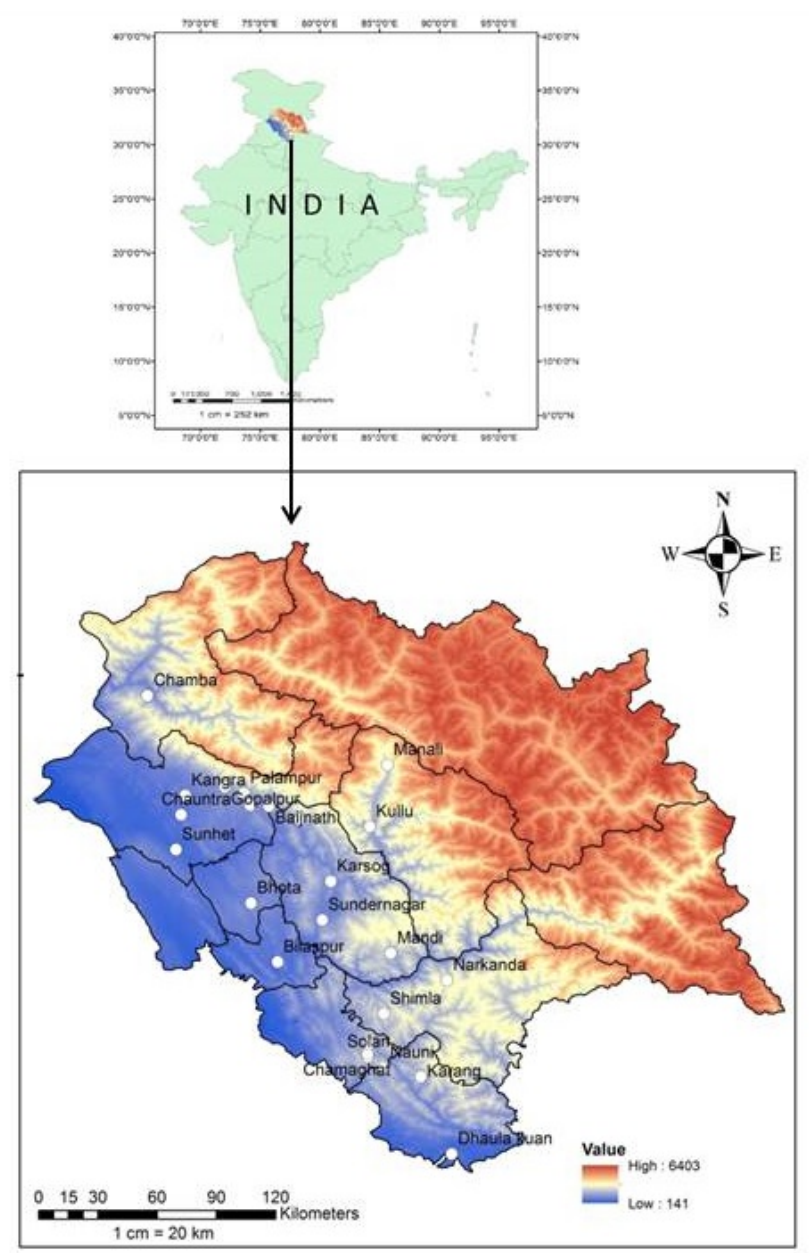

Sunder Nagar -Cercospora
Bhota-Cercospora
Solan-Cercospora, Pseudocercospora, Ramularia,
Passalora,Mycosphaerella,Teratosphaeria
Kullu-Cercospora, Pseudocercospora, Passalora,
Neopseudocercosporella
Kangra-Cercospora, Pseudocercospora,Passalora,
Stigmina,Mycosphaerella,Mycovellosiella
Chamba-Cercospora
Chauntra-Cercospora, Pseudocercospora, Sirosporiur
Shimla-Cercospora, Pseudocercospora,
Ramularia,Sirosporium, Distomycovellosiella
Baijnath-Cercospora
Bilaspur-Cercospora, Ramularia
Chamaghat-Cercospora
Manali-Cercospora, Pseudocercospor
Kandaghat-Cercospora
Karsog-Nothopassalora
Mandi-Passalora,Neocercosporidium, Rosisphaerella
Nauni-Cercospora, Pseudocercospora, Stigmina
Palampur-Cercospora, Pseudocercospora, Stigmina
Joginder nagar-Pseudocercospora, Passalora
Sunhet- Pseudocercospora
Dhaula Kuan- Pseudocercospora
Panchrukhi- Pseudocercospora
Narkanda-Ramularia
Karang-Passalora
Gopalpur-Mycosphaerella,Cercosporella

Fig. 1 - Map of Himachal Pradesh showing diversity and distribution of cercosporoid fungi.

This checklist of cercosporoid fungi reported from Himachal Pradesh consists of 15 genera recorded on 118 plant species belonging to 46 plant hosts of family. Highest numbers of cercosporoid fungi were recorded on plant hosts of family Solanaceae (12 species) followed by Fabaceae (10 species), Asteraceae \& Rosaceae (6 species each), Amaranthaceae (5 species), Malvaceae \& Smilaceae (4 species each), Acanthaceae, Poaceae, Polygonaceae \& Ranunculaceae (3 species each), while rest of families were found associated with 1-2 speices cercosporoid fungi.

After compilation of the literature it was noticed that most of the records of cercosporioid fungi were reported from Solan (53), Kangra (24) followed by Mandi (17), Shimla (11), Kullu (8), Bilaspur \& Sirmaur (2 each) and Chamba \& Hamirpur (1 each). No records were found reported from Kinnaur, Lahul \& Spiti and Una. Number of cercosporoid fungi in the most representative plant families and genera and percentages of total species number is presented in Table 1.

To understand occurrence of these fungi in the state, a location wise distribution of cercosporoid fungi is presented. Since changeable geographical and climatic conditions of the Himachal Pradesh are quite favourable for the growth and development of plant pathogenic fungi, cercosporoid fungi hold an important position among them. The present compilation reveals that 
the diversity of cercosporoid fungi in quite high in Himachal Pradesh. However, the further explorations of new collections as well as molecular characterizations will add more species to the cercosporoid species of the state.

Table 1 Number of Cercosporoid fungi in the most representative plant families and genera and percentages of total species number.

\begin{tabular}{lcclcc}
\hline \multicolumn{1}{c}{ Host Family } & $\begin{array}{c}\text { Number of } \\
\text { cercosporoid spp. }\end{array}$ & \% age & Fungal Genera & $\begin{array}{c}\text { Number of } \\
\text { fungal spp. }\end{array}$ & \% age \\
\hline Solanaceae & 12 & 11.33 & Cercospora & 48 & 46.7 \\
Fabaceae & 10 & 9.44 & Pseudocercospora & 23 & 22.4 \\
Asteraceae & 06 & 5.66 & Ramularia & 09 & 8.9 \\
Rosaceae & 06 & 5.66 & Passalora & 06 & 5.9 \\
Amaranthaceae & 05 & 4.72 & Mycosphaerella & 04 & 3.9 \\
Malvaceae & 04 & 3.77 & Stigmina & 03 & 2.9 \\
Smilaceae & 04 & 3.77 & Sirosporium & 02 & 1.9 \\
Acanthaceae & 03 & 2.83 & Cercosporella & 01 & 0.9 \\
Poaceae & 03 & 2.83 & Mycovellosiella & 01 & 0.9 \\
Polygonaceae & 03 & 2.83 & Rosisphaerella & 01 & 0.9 \\
Ranunculaceae & 03 & 2.83 & Nothopassalora & 01 & 0.9 \\
Salicaceae & 03 & 2.83 & Teratosphaeria & 01 & 0.9 \\
\hline Subtotal & $\mathbf{6 2}$ & $\mathbf{5 8 . 5 0}$ & Subtotal & 100 & 97.1 \\
\hline Other families (34) & 44 & 41.50 & Other genera (3) & 03 & 2.9 \\
\hline Total & $\mathbf{1 0 6}$ & $\mathbf{1 0 0}$ & Total & $\mathbf{1 0 3}$ & $\mathbf{1 0 0}$ \\
\hline
\end{tabular}

\section{List of cercosporoid fungi reported from Himachal Pradesh}

A complete list of cercosporoid fungal genera and their species recorded in Himachal Pradesh, their hosts and distribution is presented in alphabetical order:

Cercospora Fresen. ex Fuckel, Fungi Rhen. Exs.: No.117 (1863) [Hedwigia 2(15): 133 (1863)]

= Cercosporina Speg., Anal. Mus. nac. B. Aires, Ser. 3 13: 424 (1910) [1911]

Fig. 2

= Virgasporium Cooke, Grevillea 3(no. 28): 182 (1875)

\section{Description \& Illustration}

The genus Cercospora comprises a group of foliicolous ascomycete responsible for numerous plant diseases. It mainly causes leaf spot diseases in plants with specific symptoms in the form of round, brown, sunken spots on leaves. It is one of the well explored genera of cercosporoid fungi. The sexual stage in most of these fungi is still unknown, however, when identified, it placed in the Mycosphaerella. Microscopic characters include mycelium internal, absence of stromata or very small if present; acicular, hyaline and septate conidia with conspicuous hila produced on pigmented, unbranched, septate and smooth conidiophores with conspicuously thickened and darkened conidiogenous loci (scars) (Crous \& Braun 2003). Currently about 3168 names are available within this genus worldwide (Index Fungorum 2019 accessed on 06.12.2019).

Type species: Cercospora apii Fresen., typ. cons. prop. (Braun \& Crous 2016).

Cercospora achyranthina Thirum. \& Chupp, Mycologia 40(3): 352 (1948)

Host: On leaves of Achyranthes aspera (Amaranthaceae)

Location: Sundernagar (Mandi), H.P.

Literature: (Singh et al. 1999) 
Cercospora adhatodae S. Chowdhury, Lloydia 18: 84 (1955)

Host: On leaves of Adhatoda vasica L. (Acanthaceae).

Location: Bhota (Hamirpur) H.P.

Literature: (Paul et al. 1985b)

Cercospora albiziae A.K. Kar \& M. Mandal, Trans. Br. mycol. Soc. 53(3): 348 (1969)

Host: On leaves of Albizia lebbek (L.) Benth (Fabaceae)

Location: Solan (Solan) H.P.

Literature: (Bhardwaj \& Paul 1986)

Cercospora arachidicola Hori, Report Nisigahara agric. Exp. Sta., Tokyo: 26 (1917)

Host: On leaves of Arachis hypogaea L. (Fabaceae)

Location: Solan (Solan) H.P.

Literature: (Paul \& Sharma 1999)

Cercospora asparagi Sacc., Michelia 1(no. 1): 88 (1877)

= Cercospora asparagicola (Speg.) Vassiljevsky, in Vassiljevsky \& Karakulin, Fungi

Imperfecti Parasitici (Hyphomycetes) 1: 296 (1937)

= Cercospora caulicola G. Winter, Hedwigia 24(5): 203 (1885)

= Cercosporina asparagicola Speg., Anal. Mus. nac. B. Aires, Ser. 3 13: 424 (1910) [1911]

Host: Leaves \& stem of Asparagus officinalis L. (Asparagaceae)

Location: Solan (Solan), H.P.

Literature: (Sydow \& Butler 1907, Sydow \& Mcrae 1929)

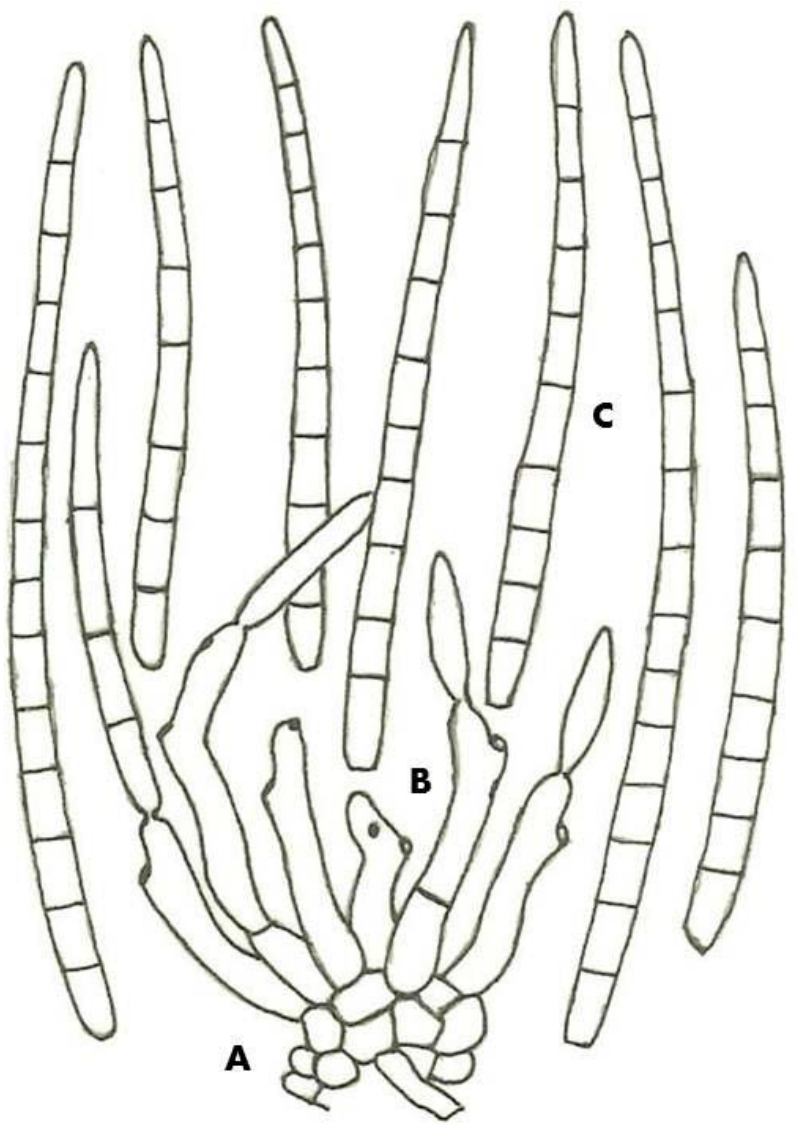

Fig. 2 - Cercospora apii. A Conidiophore fascicles. B Conidiophores. C Conidia. (Seifert et al. 2011)

Cercospora beticola Sacc., Nuovo G. bot. ital. 8(2): 189 (1876) 
= Cercospora beticola var. poonensis Chidd., Sydowia 13(1-6): 153 (1959)

= Cercosporina beticola (Sacc.) Nakata, T. Nakajima \& K. Katimoto, Rep. Agric. Korea: 6 (1915)

Host: Living leaves of Beta vulgais L. var. mangel (Amaranthaceae).

Location: Katrain (Kullu) and Kangra (Kangra), H.P.

Literature: (Lall et al. 1962)

Cercospora bombacicola Munjal, Lall \& Chona, Indian Phytopath. 13(2): 144 (1961) [1960]

Host: leaves of Salmalia malabarica (DC.) Schott \& Endl. (Malvaceae).

Location: Chamba (Chamba), H.P.

Literature: (Munjal et al. 1960)

Cercospora brachiate Ellis \& Everh., J. Mycol. 4(1): 5 (1888)

Host: On leaves of Amaranthus sp. (Amaranthaceae)

Location: Solan (Solan) H.P.

Literature: (Paul et al. 1985b)

Cercospora canescens Ellis \& G. Martin, Am. Nat. 16(12): 1003 (1882)

$=$ Cercospora vignicaulis Tehon, Mycologia 29(4): 436 (1937)

= Cercosporiopsis canescens (Ellis \& G. Martin) Miura, Flora of Manchuria and East Mongolia, III Cryptogams, Fungi (Industr. Contr. S. Manch. Rly 27): 527 (1928)

Host: On leaves of Vigna sinensis (L.) Savi Ex Hassk., V. vexillata (L.) A. Rich. (Fabaceae)

Location: Kangra (Kangra) and Solan (Solan), H.P.

Literature: (Roy 1965, Paul et al. 1985b, 348, Paul \& Sharma 1999)

Cercospora capsici Heald \& F.A. Wolf, Mycologia 3(1): 15 (1911)

Host: On leaves of Capsicum annuum L. (Solanaceae)

Location: Chauntra (Mandi) H.P.

Literature: (Paul et al. 1985a)

Cercospora citrullina Cooke, Grevillea 12(61): 31 (1883)

Host: Leaves of Rumex orientalis and R. nepalensis (Polygoniaceae); Cucurbita moschata Duch. (Cucurbitaceae); Cucumis sativus L. (Cucurbitaceae); Momordica charantia L. (Cucurbitaceae)

Location: Shimla (Shimla) and Solan (Solan), H.P.

Literature: (Padwick 1946, Paul et al. 1985a)

Cercospora daturicola (Speg.) Vassiljevsky, in Vassiljevsky \& Karakulin, Fungi Imperfecti Parasitici (Hyphomycetes) 1: 347 (1937)

Host: On leaves of Datura metel L. \& Datura stramonium L. (Solanaceae)

Location: Solan (Solan) H.P.

Literature: (Paul et al. 1985b)

Cercospora dianthi A.S. Mull. \& Chupp, Arq. Inst. Biol. Veget. Rio de Janeiro 3: 93 (1936)

Host: On leaves of Dicliptera roxburghiana Nees. (Acanthaceae)

Location: Solan (Solan) H.P.

Literature: (Chandel 1998)

Cercospora digitalis P.K. Chi \& C.K. Pai [as ‘digitalidis'], Acta phytotax. sin. 10: 113 (1966)

Host: On leaves of Digitalis purpurea L. (Plantaginaceae)

Location: Solan (Solan) H.P.

Literature: (Paul et al. 1985b) 
Cercospora doryalidis Chupp \& Doidge, Bothalia 4: 885 (1948)

Host: On leaves of Flacourtia indica (Burm. f.) Merr. (Salicaceae)

Location: Bilaspur (Bilaspur) H.P.

Literature: (Paul et al. 1985b)

Cercospora duddiae Welles, Phytopathology 13: 364 (1923)

Host: On leaves of Allium tuberosum Rottler ex Spreng. (Amaryllidaceae).

Location: Solan (Solan) H.P.

Literature: (Paul et al. 1985b)

Cercospora eugeniae Chupp, Monograph of Cercospora: 406 (1954)

Host: On leaves of Syzigium cumini (L.) Skeels. (Myrtaceae)

Location: Baijnath (Kangra) H.P.

Literature: (Singh et al. 1999)

Cercospora fukushiana (Matsuura) W. Yamam., Journal of the Society of Tropical Agriculture, Formosa 6: 601 (1934)

Host: On leaves of Impatiens balsamina L. \& Impatiens gigantea Edgew. (Balsaminaceae)

Location: Solan (Solan) H.P.

Literature: (Paul et al. 1985b)

Cercospora geranii Kellerm. \& Swingle, J. Mycol. 5(2): 74 (1889)

Host: On leaves of Geranium sp. (Geraniaceae)

Location: Kangra (Kangra) H.P.

Literature: (Lall et al. 1961)

Cercospora gerberae Chupp \& Viégas, in Viegas, Boln da Soc. Brasil de Agron. 8: 27 (1945)

Host: On leaves of Gerbera sp. (Asteraceae)

Location: Baijnath (Kangra) H.P.

Literature: (Singh et al. 1999)

Cercospora helianthicola Chupp \& Viégas, in Viegas, Boln da Soc. Brasil de Agron. 8: 29 (1945)

Host: On leaves of Helianthus annuus L. (Asteraceae)

Location: Solan (Solan) H.P.

Literature: (Singh et al. 1999)

Cercospora hydrangea Ellis \& Everh., in Atkinson, J. Elisha Mitchell scient. Soc. 8(2): 52 (1892)

Host: On leaves of Hydrangea sp. (Hydrangeaceae)

Location: Chambaghat (Solan) H.P.

Literature: (Paul et al. 1985c)

Cercospora justiciicola F.L. Tai, Lloydia 11: 47 (1948)

Host: On leaves of Justicia sp. (Acanthaceae)

Location: Chambaghat (Solan) H.P.

Literature: (Paul et al. 1985c)

Cercospora kikuchii (Tak. Matsumoto \& Tomoy.) M.W. Gardner, Proc. natn. Acad. Sci. India, Sect. B, Biol. Sci. 36: 12 (1927) [1926]

= Cercosporina kikuchii Tak. Matsumoto \& Tomoy., Ann. phytopath. Soc. Japan 1(6): 1(1925)

Host: seeds of Dolichos biflorus L. (Fabaceae) \& Glycine max (L.) Merr. (Fabaceae).

Location: Solan (Solan) and Kangra (Kangra), H.P.

Literature: (Saharan 1979, Paul 1989) 
Cercospora leucosticte Ellis \& Everh., J. Mycol. 4(6): 53 (1888)

Host: On leaves of Melia azadirachta L. (Meliaceae)

Location: Sundernagar (Mandi) H.P.

Literature: (Singh et al. 1999)

Cercospora lygodiicola Lall, J.N. Kapoor \& Munjal, Indian Phytopath. 17: 181 (1964)

Host: Leaves of Lygodium japonicum (Thunb.) SW. (Lygodiaceae)

Location: Kullu \& Manali (Kullu), H.P.

Literature: (Lall et al. 1964)

Cercospora megaspermae Bhardwaj \& Sharma, Indian Foreser 120(6): 545 (1994)

Host: Leaves of Pistacia integrrima L. (Anacardiaceae).

Location: Solan (Solan), H.P.

Literature: (Prakasam 1991, Bhardwaj \& Sharma 1994)

Cercospora melongenae Welles, Phytopathology 12: 63 (1922)

= Cercosporina melongenae (Welles) Hara, J. agric. Soc. Japan 32(no. 364): 46 (1928)

Host: On leaves of Solanum melongena L. (Solanaceae)

Location: Solan (Solan) H.P.

Literature: (Paul \& Sharma 1999)

Cercospora melothriae Sawada, Rep.Gov. Res. Ins., Formosa 86: 173 (1943)

Host: On leaves of Melothria heterophylla (Lour.) Cogn. (Cucurbitaceae)

Location: Kandaghat (Solan) H.P.

Literature: (Paul et al. 1985c)

Cercospora mitteriana Syd., Annls mycol. 35(3/4): 240 (1937)

Host: On leaves of Dodonaea viscosa (L.) Jacq. (Sapindaceae)

Location: Solan (Solan) H.P.

Literature: (Paul \& Sharma 1999)

Cercospora nebulosi Sacc., Nuovo G. bot. ital. 8(2): 189 (1876)

Host: On leaves of Althea rosea L. (Malvaceae)

Location: Solan (Solan) H.P.

Literature: (Paul et al. 1985c)

Cercospora nigri Tharp, Mycologia 9(2): 112 (1917)

Host: on leaves of Solanum nigrum L. (Solanaceae)

Location: Solan (Solan), H.P.

Literature: (Bhardwaj \& Paul 1987, Philip et al. 1994a, b)

Cercospora oplismeni Lall, H.S. Gill \& Munjal, Indian Phytopath. 14(2): 117 (1962) [1961]

Host: Leaves of Oplismenus sp. (Poaceae)

Location: Shimla (Shimla), H.P.

Literature: (Lall et al. 1961)

Cercospora oxyphylli Pavgi \& U.P. Singh, Sydowia 24(1-6): 118 (1971) [1970]

Host: On leaves of Zanthoxylum alatum Wall. (Rutaceae)

Location: Solan (Solan) H.P.

Literature: (Paul et al. 1985c) 
Cercospora persicae Sacc., Qd Nat.: 189 (1876)

Host: Leaves of Prunus persica (L.) Batsch (Rosaceae )

Location: Solan (Solan), H.P.

Literature: (Sharma \& Paul 1986)

Cercospora physalidicola Ellis \& Barthol., Erythea 4: 28 (1896)

Host: On leaves of Physalis minima L. (Solanaceae)

Location: Nauni (Solan) H.P.

Literature: (Paul et al. 1985c)

Cercospora physalidis Ellis, Am. Nat. 16: 810 (1882)

= Cercosporina physalidis (Ellis) Miura, Flora of Manchuria and East Mongolia, III Cryptogams, Fungi (Industr. Contr. S. Manch. Rly 27): 525 (1928)

Host: On leaves of Smilax aspera L. (Solanaceae)

Location: Solan (Solan) H.P.

Literature: (Paul et al. 1985c)

Cercospora pisi-sativi J.A. Stev., Rep. P.Rico insul. agric. Exp. Station, 1917-18: 138 (1919)

Host: On leaves of Pisum sativum L. (Fabaceae)

Location: Palampur (Kangra) H.P.

Literature: (Singh et al. 1999)

Cercospora ricinella Sacc. \& Berl., Atti Inst. Veneto Sci. lett., ed Arti, Sér. 6 3: 721 (1885)

Synonymy:

= Cercospora albidomaculans G. Winter, Hedwigia 24(5): 202 (1885)

= Cercospora ricini Speg., Anal. Mus. nac. Hist. nat. B. Aires 6: 343 (1898) [1899]

= Cercosporina ricinella (Sacc. \& Berl.) Speg., Anal. Mus. nac. B. Aires, Ser. 3 13: 429

(1910) [1911]

Host: On leaves of Ricinus communis L. (Euphorbiaceae)

Location: Mandi (Mandi) H.P.

Literature: (Singh et al. 1999)

Cercospora sesami Zimm., Ber. über Land. und Forstwirth. Deutsch-Ostafrica: 29 (1904)

Host: On leaves of Sesamum indicum L. (Pedaliaceae)

Location: Solan (Solan) H.P.

Literature: (Munjal \& Sharma 1976)

Cercospora solani Thüm., Hedwigia 19: 135 (1880)

Host: On leaves of Solanum nigrum L. (Solanaceae).

Location: Solan (Solan) H.P.

Literature: (Paul et al. 1985c)

Cercospora solanicola G.F. Atk., J. Elisha Mitchell scient. Soc. 8(2): 53 (1892)

Host: Tuber of Solanum tuberosum L. (potato) in storage (Solanaceae)

Location: Shimla (Shimla), H.P.

Literature: (Rai 1983)

Cercospora solani-tuberosi Thirum., Amer. Potato J. 30: 96 (1953)

Host: On leaves of Solanum tuberosum L. (Solanaceae)

Location: Solan (Solan) H.P.

Literature: (Paul et al. 1985c) 
Cercospora sonchi Chupp, Monograph of Cercospora: 159 (1954)

= Cercospora sonchi var. taraxaci Govindu \& Thirum., Sydowia 18(1-6): 21 (1965) [1964]

Host: On leaves of Sonchus oleraceus L. (Asteraceae)

Location: Solan (Solan) H.P.

Literature: (Paul et al. 1985c)

Cercospora sorghi Ellis \& Everh., J. Mycol. 3(2): 15 (1887)

$=$ Cercospora sorghi $f$. maydis (Ellis \& Everh.) Sacc., Syll. fung. (Abellini) 10: 656 (1892)

$=$ Cercospora sorghi var. ciccaronei (N. Pons) U. Braun, Schlechtendalia 5: 48 (2000)

= Cercospora sorghi var. maydis Ellis \& Everh., J. Mycol. 3(2): 15 (1887)

= Phaeoramularia ciccaronei N. Pons, Fitopatol. Venez. 6(1): 2 (1993)

Host: Leaves of Zea mays L. (Poaceae).

Location: Kullu (Kullu), H.P.

Literature: (Lall et al. 1963)

Cercospora tageticola Ellis \& Everh., J. Mycol. 8(2): 72 (1902)

Host: On leaves of Tagetes patula L. (Asteraceae)

Location: Solan (Solan) H.P.

Literature: (Munjal \& Sharma 1976)

Cercospora traversoana Sacc. [as 'traversiana'], Annls mycol. 2(1): 18 (1904)

Host: On leaves of Trigonella foenum-graecum L. (Fabaceae).

Location: Kangra (Kangra) H.P.

Literature: (Singh et al. 1999)

Cercospora violae Sacc., Nuovo G. bot. ital. 8(2): 187 (1876)

= Cercospora violae var. minima Gonz. Frag. \& Cif., Boln Real Soc. Españ. Hist. Nat.,

Biologica 27: 278 (1927)

= Cercospora violae var. minor Rota-Rossi, Atti Ist. bot. R. Univ. Pavia, 2 Sér. 13: 199 (1914)

Host: On leaves of Viola serpens Wall. (Violaceae)

Location: JoginderNagar (Mandi) H.P.

Literature: (Singh et al. 1999)

Cercospora zeae-maydis Tehon \& E.Y. Daniels, Mycologia 17(6): 248 (1925)

Host: Seeds of Zea mays L. (Poaceae).

Location: Solan (Solan), H.P.

Literature: (Paul et al. 1990, Paul et al. 1985b)

Cercospora zinnia Ellis \& G. Martin, J. Mycol. 1(1): 20 (1885)

Host: On leaves of Zinnia elegans Jacq. (Asteraceae).

Location: Solan (Solan) H.P.

Literature: (Munjal \& Sharma 1976, Paul \& Bhardwaj 1986b)

Cercosporella Sacc., Michelia 2 (6): 20 (1880)

Fig. 3

\section{Description \& Illustration}

Cercosporella (Mycosphaerellaceae) is a group of phytopathogenic cercosporoid fungi, mostly causing leaf spot diseases. The genus is lacking of pigment in the conidiophores and spores, which distingiuinshed it from the genus Cercospora and other members of the family. Primary mycelium is internal, and stroma present. Conidiophores fasciculate, simple or branched, smooth, emerging through the stromata. Conidiogenous cells integrated, terminal, polyblastic, sympodial, 
conspicuously cicatrized, and geniculate at old conidial scars. Secondary mycelium rare consisting of external repent, hyaline hyphae which bear secondary conidiophores as lateral branches. Conidial scars conspicuous, thickened, colourless and refractive, the thickening extending beyond the areas occupied by the base of the conidium. A minute papilla in the centre of the old scar and a minute frill at the apex of the conidiogenous cell are often seen. Conidia colourless or faintly greenish, smooth, thin-walled, usually subcylindric and slightly obclavate, sometimes fusiform, 1many septate, with an obtuse apex and narrowed at the base. Hilum slightly convex, colourless, refractive, slightly thickened but often not very conspicuous (Deighton 1973). Cercosporella species with secondary mycelium are placed in a speatrate subgenus namely; 1 . Cercosporella subgen. Cercosporella: Mycelium internal, secondary mycelium lacking. Conidiophors fasciculate, rarely solitary, emerging through stromata, rarely erumpt through cuticle.

2. Cercosporella subgen. Pseudovellosiella: Promary mycelium internal, secondary mycelium external, superficial. Conidiophors fasciculate and/or solitary, arising from creeping secondary hyphae (Braun 1995). There are 274 epithets reported worldwide in the genus Cercosporella (Index Fungorum 2019 accessed on 06.12.2019) nearly on all plant families.

Type species: Cercosporella cana (Sacc.) Sacc., Michelia 2(no. 6): 20 (1880)

Cercosporella persicae (Sacc.) Sacc., Michelia 2 (6): 20 (1880)

Host: Leaves of Prunus armeniaca (Rosaceae)

Location: Gopalpur (Kangra), H.P.,

Literature: (Sohi et al. 1964)

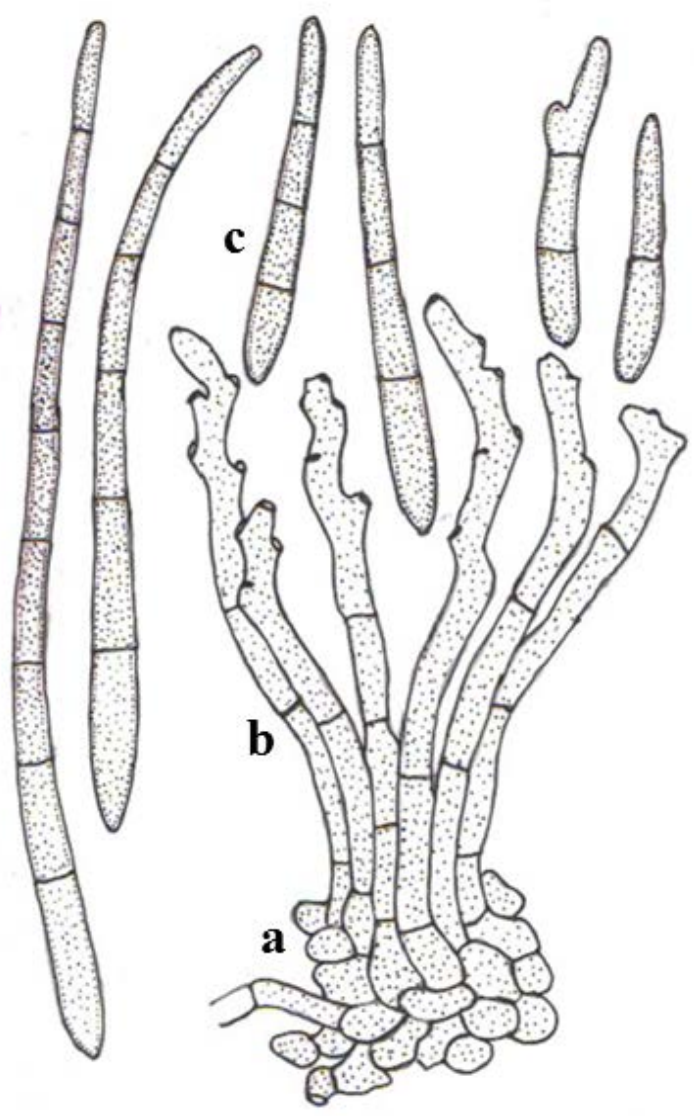

Fig. 3 - Cercosporella virgaureae. A Conidiophore fascicles. B Conidiophores. C Conidia. (Seifert et al. 2011)

Distomycovellosiella U. Braun, C. Nakash., Videira \& Crous, Studies in Mycology 87: 330 (2017) 


\section{Description \& Illustration}

The genus Distomycovellosiella comprises a group of plant pathogenic fungi producing caespituli hypophyllous, pale brown or olivaceous, floccose colonies in infected substrate. Mycelium in host tissue hyaline and internal, produce pale brown to brown external hyphae. Stromata either absent or small, if present. Conidiophores arising solitary from external hyphae as straight to geniculate, simple, sometimes branched or through stomata as loose to dense coremioid fascicles. Conidia pale brown to pale olivaceous, smooth to verruculose, ovoid, obovoid, obclavate, clavate, cylindrical, fusiform, straight or slightly curved, aseptate, borne unbranched or branched chains (catenate) (Videira et al. 2017).

Type species: Distomycovellosiella brachycarpa (Syd.) U. Braun, C. Nakash., Videira \& Crous 2017

Distomycovellosiella brachycarpa (Syd.) U. Braun, C. Nakash., Videira \& Crous, in Videira, Groenewald, Nakashima, Braun, Barreto, de Wit \& Crous, Stud. Mycol. 87: 330 (2017)

= Cercospora brachycarpa Syd., Annls mycol. 28(1/2): 207 (1930)

= Mycovellosiella brachycarpa (Syd.) Deighton, Mycol. Pap. 137: 8 (1974)

= Passalora brachycarpa (Syd.) U. Braun \& Crous, in Crous \& Braun, CBS Diversity

Ser. (Utrecht) 1: 87 (2003)

= Cercospora jaguarensis Chupp \& A.S. Mull., Bol. Soc. Venez. Ci. Nat., 8:48. 1942.

= Cladosporium solanicola Viegas, Bragantia 6: 368. 1946.

= Mycovellosiella solanicola (Viegas) Munt-Cvetk. Lilloa 30: 178. 1960.

Host: Leaves of Solanum tuberosum L. (Solanaceae).

Location: Shimla (Shimla), H.P.

Literature: (Kamal 2010)

Mycosphaerella Johanson, Öfvers. K. Svensk. Vetensk.-Akad. Förhandl. 41(no. 9): 163 (1884)

\section{Description \& Illustration}

Mycosphaerella is one of the largest genera of plant pathogenic fungi. Mycelium is internal and external; brached, septate, olivaceous to brown, thinwalled, smooth. Superficial (external) hyphae are generally emerging through stomata. Conidiophores are solitary or form loose to fairly dense fascicles. Single conidiophores arising from superficial hyphae, erect, straight, subcylindricalconical to geniculate-sinuous, unbranched, 0-3-septate, olivaceous brown to brown, thin-walled and smooth. Conidiogenous cells are integrated, terminal or conidiophores reduced to conidiogenous cells and bearing solitary, obclavate-cylindrical, straight to curved or even sigmoid conidia. The conidia are generally 2-12-septate, subhyaline to pale olivaceous brown, thin-walled, smooth, apex obtuse to subacute, base short obconically truncate with slightly thickened and darkened hila (Sivanesan 1984, Crous et al. 2000). There are 1787 epithets reported worldwide in the genus Mycosphaerella (Index Fungorum 2019 accessed on 06.12. 2019).

Type species: Mycosphaerella punctiformis (Pers.) Starbäck 1889

Mycosphaerella cerasella Aderh., Ber. dt. bot. Ges. 18: 246 (1900)

= Cercospora cerasella Sacc., Michelia 1(no. 2): 266 (1878)

= Cercospora circumscissa Sacc., Fungi venet. nov. vel. Crit., Sér. 5: 189 (1878)

$=$ Cercosporella cerasella (Sacc.) Jacz., Yearbook of Information Concerning Diseases and

Injuries of Cultivated and Wild Economic Plants: [1] (1903)

= Passalora circumscissa (Sacc.) U. Braun [as 'circumcissa'], Mycotaxon 55: 230 (1995)

= Pseudocercospora circumscissa (Sacc.) Y.L. Guo \& X.J. Liu, Mycosystema 2: 231 (1989)

= Sphaerella cerasella (Aderh.) Sacc. \& P. Syd., Syll. fung. (Abellini) 16: 469 (1902)

Host: On leaves of Prunus persica (L.) Batsch (Rosaceae) 
Location: Solan (Solan) H.P.

Literature: (Paul \& Sharma 1999)

Mycosphaerella cruenta Latham, Mycologia 26(6): 525 (1934)

= Cercospora cruenta Sacc., Michelia 2(no. 6): 149 (1880)

= Pseudocercospora cruenta (Sacc.) Deighton, Mycol. Pap. 140: 142 (1976)

Host: Leaves of Dolichos biflorus L. (Fabaceae); Populus yunnanensis Dode. (Salicaceae)

Location: Solan (Solan) and Kangra (Kangra), H.P.

Literature: (Paul \& Sharma 1999, Kamal 2010)

Mycosphaerella pruni-persicae Deighton, Trans. Br. mycol. Soc. 50(2): 328 (1967)

= Cercospora persicae Sacc. [as 'persica'], Qd Nat.: 189 (1876)

= Cercosporella persicae (Sacc.) Sacc. [as 'persica'], Michelia 2(no. 6): 20 (1880)

= Clasterosporium persicae (Sacc.) Tsuji, Ann. phytopath. Soc. Japan 1(2): 33 (1919)

= Fusarium persicae (Sacc.) G.F. Atk., J. Elisha Mitchell scient. Soc. 8(2): 41 (1892)

= Miuraea persicae (Sacc.) Hara, Byogaichu-Hoten (Manual of Pests and Diseases): 224

= Mycosphaerella persicae B.B. Higgins \& F.A. Wolf [as 'persica'], Phytopathology 27: 695 (1937)

Host: Leaves of Prunus armeniaca L. (Rosaceae)

Location: Gopalpur (Kangra), H.P.

Literature: (Sohi et al. 1964, Sohi \& Gupta 1966)

Mycovellosiella Rangel, Archos Jard. bot., Rio de J. 2: 71 (1917)

= Walkeromyces Thaung, Trans. Br. mycol. Soc. 66(2): 213 (1976)

Fig. 4

\section{Description \& Illustration}

Mycovellosiella is a genus of plant pathogen fungi infecting wide range of plants. These symptoms of these fungi on infected plants appear as effuse, greyish or olivaceous brown, velvety colonies on leaves with internal primary and abundant secondary hyaline to pigmented mycelium. Stromata absent or small/ rudimentary. Conidiophores solitary, arising from superficial hyphae, as well as fasciculate, subhyaline to pigmented, simple or branched. Conidiogenous cells integrated, terminal, intercalary or pleurogenous, polyblastic, sympodial, cicatrized, conidial scars conspicuous, thickened and darkened. Conidia solitary or catenate branched, amerosporous to scolecosporous, acropleurogenous, simple, cylindrical with rounded ends, narrowly ellipsoidal, fusiform to obclavate, hyaline to pigmented, smooth, 0-3 or more septate. The genus consists in total 201 records worldwide (Index Fungorum 2019 accessed on 06.12.2019).

Type species: Mycovellosiella cajani (Henn.) Rangel ex Trotter, Sylloge Fungorum 25: 942 (1931)

Mycovellosiella bellynckii (Westend.) Constant., Cryptog. Mycol. 3(1): 67 (1982)

= Cercospora bellynckii (Westend.) Niessl, Hedwigia 15: 1 (1876)

= Cercosporidium bellynckii (Westend.) X.J. Liu \& Y.L. Guo, Acta Mycol. Sin. 1(2): 93 (1982)

= Cladosporium bellynckii Westend., Bull. Acad. R. Sci. Belg., Cl. Sci. 21(2): 240 (1854)

= Passalora bellynckii (Westend.) U. Braun, Mycotaxon 55: 228 (1995)

= Passalora bellynckii (Westend.) Poonam Srivast., Journal of Living World 1(2): 115 (1994)

Host: Leaves of Beta cicla (L.) Pers. (Amaranthaceae)

Location: Kangra (Kangra), H.P.

Literature: (Lall et al. 1961) 


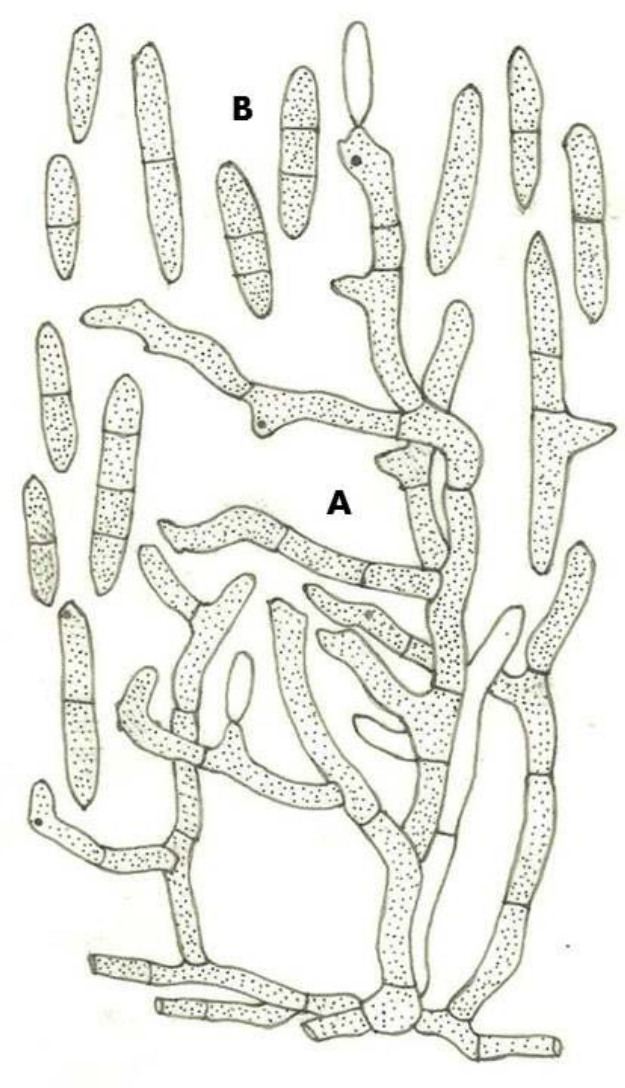

Fig. 4 - Mycovellosiella cajani. A Conidiophore. B Conidia. (Seifert et al. 2011)

Neocercosporidium Videira \& Crous, Studies in Mycology 87: 325 (2017)

\section{Description \& Illustration}

Neocercosporidium is a genus of phytopathogenic fungi appears as amphigenous, punctiform, scattered to dense, dark brown to blackish spots. These fungi possess internal and external, branched, septate, subhyaline to medium olivaceous brown, thin-walled, smooth mycelium. Conidiophores arising from stromata well-developed, substomatal to intraepidermal, immersed, brown to dark brown stromata, in small to large and loose to dense fascicles, when dense almost coremioid, rarely solitary, smooth, olivaceous to dark olivaceous brown throughout or paler at the tips, thin-walled, erect, straight, subcylindrical to strongly geniculate-sinuous, simple or occasionally branched, sometimes reduced to conidiogenous cells. Conidiogenous cells integrated, terminal, proliferating sympodially, occasionally percurrently. Conidia solitary, subhyaline to pale olivaceous or brownish, smooth, thin-walled, multi-septate, obclavate-cylindrical, apex obtuse to subobtuse, base round to short obconically truncate, hila slightly thickened, darkened and refractive (Videira et al. 2017). As this is newly reported genbus of cercosporid fungi, onle one record of type speies namely, Neocercosporidium smilacis is available (Index Fungorum 2019 accessed on 06.12.2019).

Type species: Neocercosporidium smilacis (Thüm.) U. Braun, C. Nakash., Videira \& Crous 2017

Neocercosporidium smilacis (Thüm.) U. Braun, C. Nakash., Videira \& Crous, in Videira, Groenewald, Nakashima, Braun, Barreto, de Wit \& Crous, Stud. Mycol. 87: 326 (2017)

= Cercospora smilacis Thüm., Inst. Rev. Sci. Litt., Coimbra 27: 14 (1879)

= Passalora smilacis (Thüm.) U. Braun, in Triebel, Arnoldia 14: 30 (1997)

Host: On leaves of Smilax aspera L. (Smilacaceae).

Location: Mandi (Mandi) H.P.

Literature: (Paul \& Sharma 1999) 


\section{Description \& Illustration}

Neopseudocercosporella is phytopathogenic fungi causing leaf spots. These fungi hold internal, hyaline, septate, branched mycelium with stromata well developed to nearly absent. Conidiophores generally hyaline, sometimes faintly pigmented, smooth, simple, straight, slightly curved or geniculate-sinuous, usually aseptate, one- to multiseptate, emeries singly or in groups through cutitle or stomata. Conidia smooth, thin walled, pigmented to hyaline, arranged singly (solitary). Shaepe varies from subcylindrical to obclavate, with apex obtuse to subacute and base truncate (Videira et al. 2016). Only two species are recorded wiroldwide for this genus (IndexFungorum 2019 accessed on 06.12.2019).

Type species: Neopseudocercosporella capsellae (Ellis \& Everh.) Videira \& Crous 2016

Neopseudocercosporella capsellae (Ellis \& Everh.) Videira \& Crous, in Videira, Groenewald, Braun, Shin \& Crous, Stud. Mycol. 83: 86 (2016)

三Cylindrosporium capsellae Ellis \& Everh., Journal of Mycology 3 (11): 130 (1887)

$\equiv$ Cercoseptoria capsellae (Ellis \& Everh.) H.C. Greene, Transactions of the Wisconsin

Academy of Science 47: 127 (1959)

E Pseudocercosporella capsellae (Ellis \& Everh.) Deighton, Mycological Papers 133: 42

ECercoseptoria capsellae (Ellis \& Everh.) Arx, Proceedings van de Koninklijke Nederlandse Akademie van Wetenschappen Section C 86 (1): 35 (1983)

三Pseudocercospora capsellae (Ellis \& Everh.) M.J. Morris \& Crous, South African Journal of Botany 60 (6): 325 (1994)

= Cylindrosporium brassicae Fautrey \& Roum., Revue mycol., Toulouse 13(no. 50): 61

= Cercosporella brassicae (Fautrey \& Roum.) Höhn., Annls mycol. 22(1/2): 193 (1924)

= Cercospora brassicae (Fautrey \& Roum.) Chupp, in Weiss, U.S. Dept. Agric. Handb. 165: 99 (1960)

= Cercosporella albomaculans (Ellis \& Everh.) Sacc., Syll. fung. (Abellini) 11: 606 (1895)

= Ramularia rapae Pim, J. Bot., Lond. 35: 58 (1897)

= Cercosporella rapistri Hollós, Annls hist.-nat. Mus. natn. hung. 6: 536 (1908)

= Cylindrosporium nesliae Bubák, Annls mycol. 14(5): 346 (1916)

= Cercosporella brassicae Jaap, Verh. bot. Ver. Prov. Brandenb. 64: 59 (1922)

= Cercosporella nesliana Baudyš \& Picb. [as 'nesleana'], Acta Soc. Sci. nat. moravo-siles.

1(5): 305 (1924)

= Cercospora raphanistri Baudyš \& Picb., Acta Soc. Sci. nat. moravo-siles. 1(5): 306 (1924)

= Cercosporella nesliae Dearn. \& Bisby, in Bisby, Buller \& Dearness, Fungi of Manitoba and Saskatchewan: 125 (1929)

= Cercosporella conringiae Annal., Nov. sist. Niz. Rast. 9: 195 (1972)

= Cercosporella goldbachiae Annal., Nov. sist. Niz. Rast. 9: 196 (1972)

= Cercosporella malcolmiae Annal., Nov. sist. Niz. Rast. 9: 202 (1972)

= Cercosporella litvinoviae Annal., Nov. sist. Niz. Rast. 9: 200 (1972)

Host: Leaves of Brassica rapa (Brassicaceae)

Location: Kullu (Kullu), H.P.

Literature: (Gangopadhyay \& Kapoor 1976)

Nothopassalora U. Braun, C. Nakash., Videira \& Crous, Studies in Mycology, 87: 257-421 (2017)

\section{Description \& Illustration}


Nothopassalora is a genus of hyphomycetous, phytopathogenic fungi causing leaf spot diseases on Arachis hypogaea. These fungi have internal, septate, branched, hyaline to pale brown coloured mycelium and dark, epidermal, substomatal, subglobose stromata. Conidiophores fasciculate, pale to medium brown, smooth to verruculose, simple, straight to flexuous, geniculatesinuous at the apex, multiseptate, emerging from from stromata, through stomata. Conidia pale brown to olivaceous, smooth, thin-walled, cylindrical to long-obclavate, straight or gently curved, multiseptate borne singly on conidiogenous cells. Shape of conidia reflects rounded apex and sometimes narrowing into a beak, rounded base or obconically truncate and dark thickened hila (Videira et al. 2017). As this is newly reported genus of cercosporid fungi, only one record of type speies namely, Nothopassalora personata is available (Index Fungorum 2019 accessed on 06.12.2019).

Type species: Nothopassalora personata (Berk. \& M.A. Curtis) U. Braun, C. Nakash., Videira \& Crous 2017

Nothopassalora personata (Berk. \& M.A. Curtis) U. Braun, C. Nakash., Videira \& Crous, in Videira, Groenewald, Nakashima, Braun, Barreto, de Wit \& Crous, Stud. Mycol. 87: 333 (2017)

= Cercospora personata (Berk. \& M.A. Curtis) Ellis, J. Mycol. 1(5): 63 (1885)

= Cercosporidium personatum (Berk. \& M.A. Curtis) Deighton, Mycol. Pap. 112: 71 (1967)

$=$ Cercosporiopsis personata (Berk. \& M.A. Curtis) Miura, Flora of Manchuria and East

Mongolia, III Cryptogams, Fungi (Industr. Contr. S. Manch. Rly 27): 527 (1928)

= Cladosporium personatum Berk. \& M.A. Curtis, in Berkeley, Grevillea 3 (no. 27): 106

= Passalora personata (Berk. \& M.A. Curtis) S.A. Khan \& M. Kamal, Pakist. J. scient. ind.

Res. 13: 188 (1961)

= Phaeoisariopsis personata (Berk. \& M.A. Curtis) Arx, Proc. K. Ned. Akad. Wet., Ser. C,

Biol. Med. Sci. 86(1): 43 (1983)

Host: On leaves of Arachis hypogaea L. (Fabaceae).

Location: Karsog (Mandi) H.P.

Literature: (Singh et al. 1999)

Passalora Fr., Summa veg. Scand., Sectio Post. (Stockholm): 500 (1849)

Fig. 5

= Berteromyces Cif., Sydowia 8(1-6): 267 (1954)

= Cercodeuterospora Curzi, Boll. R. Staz. Patalog. Veget. Roma 12: 3 (1932)

= Cercosporidium Earle, Muhlenbergia 1(2): 16 (1901)

= Cercosporiopsis Miura, Flora of Manchuria and East Mongolia, III Cryptogams, Fungi

(Industr. Contr. S. Manch. Rly 27): 527 (1928)

= Fulvia Cif., Atti Ist. bot. Univ. Lab. crittog. Pavia, sér. 5 10(2): 246 (1954)

= Phaeoramularia Munt.-Cvetk., Lilloa 30: 182 (1960)

= Tandonell S.S. Prasad \& R.A.B. Verma, Indian Phytopath. 23(1): 112 (1970)

= Vellosiella Rangel, Boln Agric., São Paulo 16: 151 (1915)

\section{Description \& Illustration}

The genus Passalora described by Fries in 1849 was the first genus introduced for cercosporoid hyphomycetes. The fungal species included in this genus are foliicolous, however, also caulicolous, causing leaf spots or other lesions. Mycelium superficial, internal and external, branched, septate, thin, almost smooth walled, pigmented to colourless. Stromata either absent or if well-developed, substomatal to interaepidermal, subhyaline to pigmented composed of loosly to densely aggregated swollen hyphal cells. Conidiophores solitary, loosely to densely fasciculate, arising from internal hyphae or stromata, unbranched or sometimes branched, sometimes in sporodochia or synnemata, macronematous, cylindrical, filiform to strongly geniculate-sinuous, olivaceous to medium dark brown. Conidia solitary, or catenate in simple or branched acropetal 
chains, amero- to scolecosporous, aseptate to plurieuseptate, rarely with additional distosepta, pale olivaceous to distinctly pigmented, smooth to finely rough, hila conspicuous, somewhat thickened and darkened-refractive. Over 727 epithets are available in Index fungorum for this genus (Index Fungorum 2019 accessed on 06.12.2019).

Type species: Passalora bacilligera (Mont. \& Fr.) Mont. \& Fr. In Mont., Sylloge generum specierumque cryptogamarum: 305. 1849.

Passalora avicularis (G. Winter) Crous, U. Braun \& M.J. Morris, in Morris \& Crous, S. Afr. J. Bot. 60(6): 329 (1994)

= Cercospora avicularis G. Winter, J. Mycol. 1(10): 125 (1885)

= Pseudocercospora avicularis (G. Winter) A.Z.M. Khan \& Shamsi, Bangladesh J Bot. 12(2): 108 (1983)

Host: Leaves of Polygonum aviculare L. (Polygonaceae).

Location: Kullu (Kullu) and Kangra (Kangra), H.P.

Literature: (Lall et al. 1961, Kamal 2010)
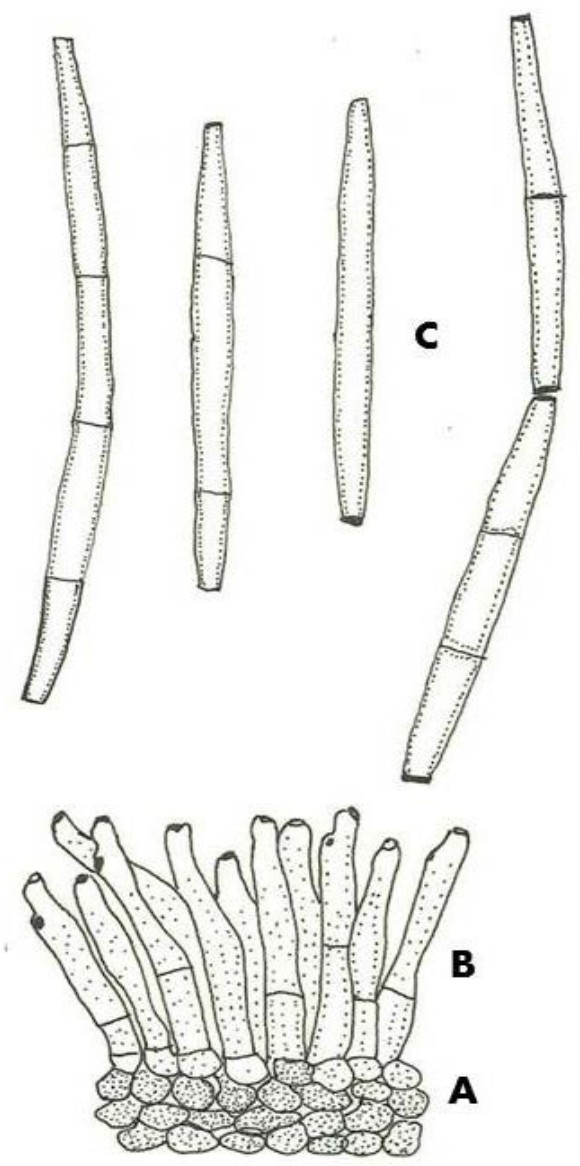

Fig. 5 - Passalora myracrodruonis. A Conidiophore fascicles. B Conidiophores. C Conidia. (Inácio \& Dianese 1999)

Passalora concors (Casp.) U. Braun \& Crous, in Crous \& Braun, CBS Diversity Ser. (Utrecht) 1: 134 (2003)

= Cercospora concors (Casp.) Sacc., Syll. fung. (Abellini) 4: 449 (1886)

= Fusisporium concors Casp., Monatsber. Königl. Preuss. Akad. Wiss. Berlin: 314 (1855)

= Mycovellosiella concors (Casp.) Deighton, Mycol. Pap. 137: 21 (1974)

Host: Leaves of Solanum tuberosum L. (Solanaceae).

Location: Solan (Solan), H.P. 
Literature: (Paul \& Sharma 1999)

Passalora lathyri-aphacae (Lall, H.S. Gill \& Munjal) U. Braun \& Crous, in Crous \& Braun, CBS Diversity Ser. (Utrecht) 1: 244 (2003)

= Cercospora lathyri-aphacae Lall, H.S. Gill \& Munjal, Indian Phytopath. 14(2): 117 (1962) [1961]

= Cercospora lathyrae-aphacae Lall, Gill \& Munjal. Leaves of Lathyrus aphaca Kangra H.P.

Host: Leaves of Lathyrus aphaca L. (Fabacee).

Location: Karang (Sirmaur), Kangra, H.P.

Literature: (Lall et al. 1961, Kamal 2010)

Passalora occidentalis (Cooke) U. Braun, Schlechtendalia 5: 70 (2000)

= Cercospora occidentalis Cooke, Hedwigia 17: 39 (1878)

= Ramularia cassiicola Heald \& F.A. Wolf [as ‘cassiaecola’], Bull. Bureau Plant Industry

U.S. Dep.Agric. 226: 101 (1912)

= Cercosporina occidentalis (Cooke) Sacc., Syll. fung. (Abellini) 25: 906 (1931)

= Phaeoramularia occidentalis (Cooke) Deighton, in Ellis, More Dematiaceous

Hyphomycetes (Kew): 322 (1976)

Host: Leaves of Cassia tora (L.) Roxb (Caesalpiniaceae).

Location: Mandi (Mandi), H.P.

Literature: (Lall et al. 1961, Kamal 2010)

Passalora pallidissima (Chupp) U. Braun, in Braun, Crous \& Nakashima, IMA Fungus 5(2): 357 (2014)

= Cercospora pallidissima Chupp, Monograph of Cercospora: 350 (1954)

= Pseudocercospora pallidissima (Chupp) Deighton, Mycol. Pap. 140: 149 (1976)

Host: Leaves of Smilax aspera L. (Smilaceae)

Location: Mandi (Mandi), H.P.

Literature: (Chona et al. 1959, Kamal 2010)

Passalora squalidula (Peck) U. Braun, in Braun \& Mel'nik, Trudy Botanicheskogo Instituta im. V.L. Komarova 20: 95 (1997)

= Cercospora squalidula Peck, Ann. Rep. N.Y. St. Mus. nat. Hist. 33: 29 (1883) [1880]

= Pseudocercospora squalidula (Peck) Y.L. Guo \& X.J. Liu, in Guo, Fungi and Lichens of Shennongjia. Mycological and Lichenological Expedition to Shennongjia (Beijing): 366 (1989)

Host: Leaves of Clemaits gouriana Roxb. ex DC. (Ranunculaceae)

Location: Mandi (Mandi), H.P.

Literature: (Lall et al. 1961, Kamal 2010)

Passalora verbeniphila (Speg.) Crous \& U. Braun, Mycotaxon 57: 310 (1996)

= Cercospora verbeniphila Speg., Boln Acad. nac. Cienc. Córdoba 29(2-3): 179 (1926)

Host: On leaves of Verbena sp. (Verbenaceae).

Location: JoginderNagar (Mandi) H.P.

Literature: (Paul et al. 1985a)

Pseudocercospora Speg., Anal. Mus. nac. B. Aires, Ser. 3 13: 437 (1910) [1911] Fig. 6 = Sawada, Report of the Department of Agriculture, Government Research Institute of

Formosa 87: 77 (1944)

= Cercocladospora G.P. Agarwal \& S.M. Singh, Proc. natn. Acad. Sci. India, Sect. B, Biol.

Sci. 42(4): 439 (1974) [1972]

= Dictyocephala A.G. Medeiros, Publicações Inst. Micol. Recife 372: 13 (1962)

= Macraea Subram., Proc. Indian Acad. Sci., Pl. Sci. 36B: 164 (1953) [1952] 
= Prathigada Subram., in Subramanian \& Ramakrishnan, Journal of Madras Univ., B 26: 366 (1956)

= Semipseudocercospora J.M. Yen, Mycotaxon 17: 361 (1983)

= Ancylospora Sawada, Report of the Department of Agriculture, Government Research Institute of Formosa 87: 77 (1944)

= Cercoseptoria Petr., Annls mycol. 23(1/2): 69 (1925)

= Ciferriella Petr., Annls mycol. 28(5/6): 409 (1930)

= Helicomina L.S. Olive, Mycologia 40(1): 16 (1948)

= Jaczewskiella Murashk., Mater. Mikol. Fitopat. Ross. 5(2): 5 (1926)

= Marcosia Syd. \& P. Syd., Annls mycol. 14(1/2): 96 (1916)

= Phaeoisariopsis Ferraris, Annls mycol. 7(3): 280 (1909)

= Prathigada Subram., in Subramanian \& Ramakrishnan, Journal of Madras Univ., B 26: 366 (1956)

= Pseudopuccinia Höhn., in Weese, Mitt. bot. Inst. tech. Hochsch. Wien 2(2): 41 (1925)

= Rhopaloconidium Petr., Sydowia 6(1-4): 300 (1952)

= Semipseudocercospora J.M. Yen, Mycotaxon 17: 361 (1983)

= Septoriopsis F. Stevens \& Dalbey, Mycologia 11(1): 4 (1918) [1919]

= Stigmina E. Bald. \& Cif., Atti Ist. bot. R. Univ. Pavia, 3 Sér. 10: 70 (1937)

\section{Description \& Illustration}

Pseudocercospora is a widely distributed genus of ascomycete fungi estimated to contain over 100 species. The genus found predominantly in tropical regions, can cause leaf spots, fruit spots, fruit rot, and blight disease. The genus is a well-known anamorph of Mycosphaerella. It can be characterized by combination of characters such as internal as well as external mycelium, conidiophores solitary, arising from superficial hyphae, lateral or terminal, fasciculate, septate or aseptate, brown to pale brown in color, synnmatous or forming distinct sporodochia; Conidia solitary, rarely catenate, scolecosporous, rarely non- scolecosporous, didymosporous phragmosporous or helocosporous, pale olivaceous tom dark brown, tranversly septate, realry with single or few oblique septa, hilium unthickened, not darkened, conidial sucession schizolytic. Over 1686 epithets are available with Index fungorum for this genus (Index Fungorum 2019 accessed on 06.12.2019).

Type species: Pseudocercospora vitis (Lev.) Speg., Annales Mus. Nac. Hist. Nat. Buenos Aires 20: 438. 1910.

Pseudocercospora abelmoschi (Ell. \& Everh.) Dieghton, Mycol. Pap. 140: 138 (1976)

= Cercospora abelmoschi Ellis \& Everh. 1893

Host: Leaves of Hibiscus rosa-sinensis L., Abelmoschus esculentus (L.) Moench. (Malvaceae)

Location: Solan (Solan) and Palampur (Kangra) H.P.

Literature: (Dhancholia \& Singh 1992, Paul \& Sharma 1999)

Pseudocercospora blumeae (Thüm.) Deighton, Mycol. Pap. 140: 140 (1976)

= Cercospora blumeae Thüm., Revue mycol., Toulouse 2(1): 38 (1880)

= Chaetotrichum blumeae (Thüm.) Petr., Sydowia 10(1-6): 129 (1957) [1956]

Host: Leaves of Buddleia sp. (Loganiaceae)

Location: Shimla (Shimla) \& Sundernagar (Mandi), H.P.

Literature: (Chona et al. 1959, Singh et al. 1999)

Pseudocercospora buddlejae (W. Yamam.) Goh \& W.H. Hsieh Trans. mycol. Soc. R.O.C. 2(2):114 (1987c)

= Cercospora buddlejae W. Yamam., Trans. Nat. Hist. Soc. Formosa 26: 279 (1936)

Host: Leaves of Buddleia sp. (Loganiaceae) 
Location: Shimla (Shimla), H.P.

Literature: (Chona et al. 1959)

Pseudocercospora cannabina (Wakef.) Deighton, Mycol. Pap. 140: 141 (1976)

= Cercospora cannabina Wakef., Bull. Misc. Inf., Kew (nos 9 \& 10): 314 (1917)

Host: On leaves of Cannabis sativa L. (Cannabaceae).

Location: Solan (Solan) H.P.

Literature: (Munjal \& Sharma 1976)

Pseudocercospora cinereae (Pavgi \& U.P. Singh) Deighton, Mycol. Pap. 140: 141 (1976)

= Cercospora cinereae Pavgi \& U.P. Singh, Mycopath. Mycol. appl. 23: 188 (1964)

= Pseudocercospora cinereae (Pavgi \& U.P. Singh) Deighton, Mycol. Pap. 140: 141 (1976)

Host: On leaves of Veronica cinerea Raf. (Plantaginaceae)

Location: Chauntra (Kangra) H.P.

Literature: (Paul et al. 1985b)

Pseudocercospora diclipterae (A.K. Kar \& M. Mandal) Deighton, Trans. Br. mycol. Soc. 88(3): 388 (1987)

= Cercospora diclipterae A.K. Kar \& M. Mandal, Trans. Br. mycol. Soc. 53(3): 337 (1969)

Host: On leaves of Dianthus caryophyllus L. (Caryophyllaceae)

Location: Solan (Solan) H.P.

Literature: (Paul et al. 1985b)

Pseudocercospora dolichi (Ellis \& Everh.) J.M. Yen, Bull. trimest. Soc. mycol. Fr. 97(3): 152 (1981)

= Cercospora dolichi Ellis \& Everh., J. Mycol. 5(2): 71 (1889)

Host: Leaves and seeds of Vigna unguiculata (L.) Walp. (Leguminosae)

Location: Solan (Solan) and Kangra (Kangra), H.P.

Literature: (Sharma 1977, Saharan 1979, Paul \& Bhardwaj 1986b, Kamal 2010)

Pseudocercospora eupatoriicola (Govindu \& Thirum.) A.Z.M. Khan \& Shamsi, Bangladesh Journal of Botany 12(2): 113 (1983)

= Cercospora eupatoriicola Govindu \& Thirum. [as ‘eupatoricola’], Sydowia 8(1-6): 225 (1954)

Host: On leaves of Ageratina adenophora (Spreng.) R.M.King \& H.Rob. (Compositae).

Location: Palampur (Kangra) H.P.

Literature: (Singh et al. 1999)

Pseudocercospora geraniicola U. Braun, Cryptog. bot. 3(2-3): 241 (1993)

= Cylindrosporium geranii Ellius \& Evern., J Mycol. 4:52, 1882

= Pseudocercospora geranii (W.B. Cooke \& Shaw) U. Braun, 19991.

= Cercospora geranii Kellern. \& Swingle, J Mycol., 5:74, 1889.

Host: Geranium sp. (Geraniaceae)

Location: Kullu (Kullu) \& Kangra (Kangra), H.P.

Literature: (Kamal 2010)

Pseudocercospora jujubae (S. Chowdhury) A.Z.M. Khan \& Shamsi, Bangladesh J Bot. 12(2): 117 (1983)

= Cercospora jujubae S. Chowdhury, Indian J Agric Res., 16:525, 1946

= Cladosporium zizyphi P. Karst., Rev. Mycol. 12 (46): 78, 1890.

Host: Leaves of Zizyphus jujube Lamk.; Z. Oenoplia (L.) Mill. (Rhamnaceae)

Location: Joginder Nagar (Mandi), H.P. 
Literature: (Chona et al. 1959, Kamal 2010)

Pseudocercospora mississippiensis (Tracy \& Earle) R.F. Castañeda \& U. Braun, Cryptog. bot. 1(1): 52 (1989)

= Cercospora mississippiensis Tracy \& Earle, Bull. Torrey bot. Club 22: 179 (1895)

Host: Leaves of Smilax sp. (Smilaceae)

Location: Kangra \& Palampur (Kangra) and Shimla (Shimla), H.P.

Literature: (Chona et al. 1959, Kamal 2010)

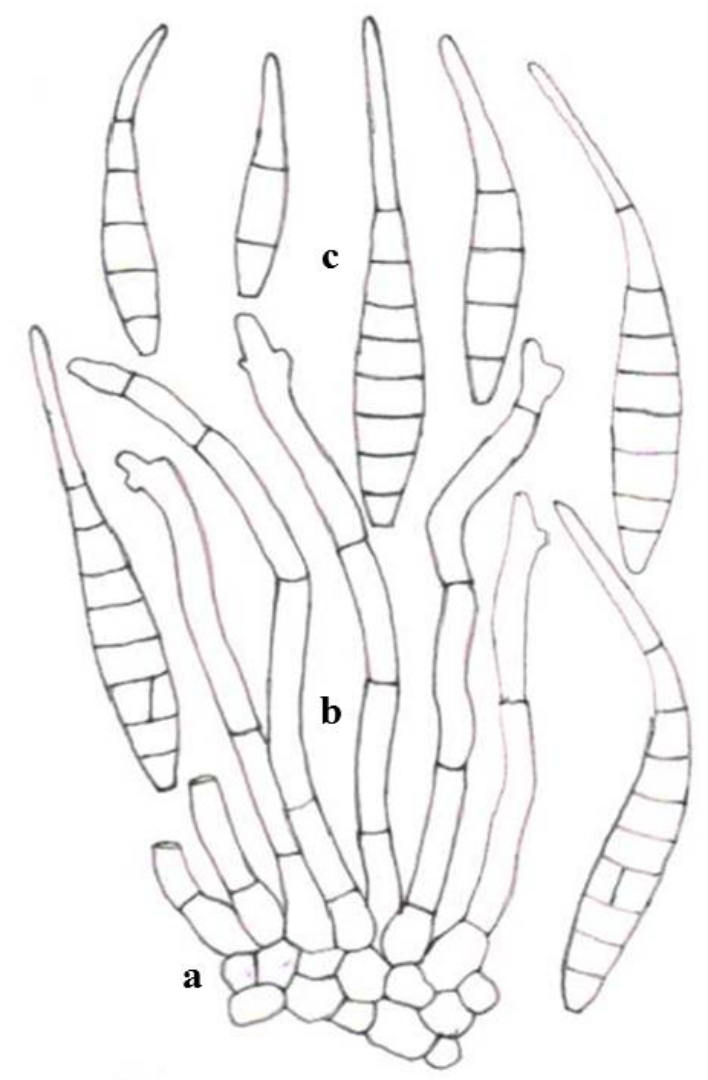

Fig. 6 - Pseudocercospora vitis. A Conidiophore fascicles. B Conidiophores. C Conidia. (Seifert et al. 2011)

Pseudocercospora mori (Hara) Deighton, Mycol. Pap. 140: 148 (1976)

= Cercospora mori Hara, Journal of the Sericultural Association of Japan 27(no. 314): 227 (1918)

Host: Leaves of Morus alba L. (Moraceae)

Location: Kangra \& Palampur (Kangra), H.P.

Literature: (Paul et al. 1986)

Pseudocercospora murrayae (A.K. Kar \& M. Mandal) Deighton, Trans. Br. mycol. Soc. 88(3): 388 (1987)

= Cercospora murrayae A.K. Kar \& M. Mandal, Trans. Br. mycol. Soc. 53(3): 357 (1969)

Host: On leaves of Murraya koenigii (L.) Sprang. (Rutaceae)

Location: Sunhet (Kangra) H.P.

Literature: (Paul et al. 1985b)

Pseudocercospora ocimicola (Petr. \& Cif.) Deighton, Mycol. Pap. 140: 149 (1976) 
= Cercospora ocimicola Petr. \& Cif., Annls mycol. 30(3/4): 324 (1932)

Host: On leaves of Ocimum tenuiflorum L. (Lamiaceae)

Location: Nauni (Solan) H.P.

Literature: (Paul et al. 1985b)

Pseudocercospora paludicola (Speg.) U. Braun, Schlechtendalia 5: 67 (2000)

= Cercospora paludicola Speg., Anal. Soc. cient. argent. 13(1): 29 (1882)

Host: Leaves of Polygonum sp. (Polygonaceae)

Location: Solan (Solan) \& Shmila (Shimla), H.P.

Literature: (Munjal et al. 1960, Kamal 2010)

Pseudocercospora platanigena Videira \& Crous, in Videira, Groenewald, Nakashima, Braun, Barreto, de Wit \& Crous, Stud. Mycol. 87: 410 (2017)

Host: Leaves of Platanus orientalis L. (Platanaceae)

Location: Manali (Kullu), H.P.

Literature: (Bakshi et al. 1970)

Pseudocercospora plumeriae (Chupp) Tak. Kobay., Nishij. \& C. Nakash., Mycoscience 39(2): 188 (1998)

= Cercospora plumeriae Chupp, Monograph of Cercospora: 49 (1954)

Host: Leaves of Plumeria tomentosa (Apocynaceae).

Location: Dhaulakuan (Sirmour), H.P.

Literature: (Sohi \& Gupta 1966, Kamal 2010)

Pseudocercospora punicae (Henn.) Deighton, Mycol. Pap. 140: 151 (1976)

= Cercospora punicae Henn., Bot. Jb. 37: 165 (1905)

Host: Leaves of Punica granatum L. (Lythraceae).

Location: Solan (Solan), H.P.

Literature: (Paul et al. 1985a)

Pseudocercospora riachueli (Speg.) Deighton, Mycol. Pap. 140: 129 (1976)

= Cercospora horiana Togashi \& Katsuki, Sci. Rep. Yokohama Natl. Univ., Sect. 2 1: 4 (1952)

= Cercospora riachueli Speg., Anal. Soc. cient. argent. 10(1): 33 (1880)

= Pseudocercospora riachueli var. horiana (Togashi \& Katsuki) U. Braun \& Crous, in Crous

\& Braun, CBS Diversity Ser. (Utrecht) 1: 354 (2003)

Host: Living leaves of Vitis vinifera L. (Vitaceae).

Location: Kullu (Kullu), H.P.

Literature: (Munjal \& Seth 1966, Munjal \& Sharma 1975, Kamal 2010)

Pseudocercospora salicina (Ellis \& Everh.) Deighton, Mycol. Pap. 140: 94 (1976)

Host: Leaves of Salix tetrasperma Roxb. (Salicaceae)

Location: Panchrukhi (Kangra), H.P.

Literature: (Paul \& Sharma 1999)

Pseudocercospora subsessilis (Syd. \& P. Syd.) Deighton, Mycol. Pap. 140: 154 (1976)

= Cercospora subsessilis Syd. \& P. Syd., Annls mycol. 11(4): 329 (1913)

= Cercospora subsessilis var. azadirachtae R.C. Srivast. [as ‘azadirachtii'], Zentbl. Bakt.

ParasitKde, Abt. II 135(6): 559 (1980)

= Cercosporina subsessilis (Syd. \& P. Syd.) Sacc., Syll. fung. (Abellini) 25: 911 (1931)

Host: On leaves of Azadirachta indica A. Juss. (Meliaceae)

Location: Solan (Solan) H.P. 
Literature: (Paul \& Sharma 1999)

Pseudocercospora sydowiana (Chupp) U. Braun \& Crous, in Braun, Schlechtendalia 2: 25 (1999)

= Cercospora sydowiana Chupp, Monograph of Cercospora: 363 (1954)

= Cercospora woodfordiae Syd., in Sydow \& McRae, Annals Cryptog. Exot. 2(3-4): 271

(1929) [1930]

= Pseudocercospora woodfordiae X.J. Liu \& Y.L. Guo, Acta Mycol. Sin. 12(1): 32 (1993)

Host: On leaves of Woodfordia floribunda (L.) Kurz (Lythraceae)

Location: Solan (Solan) H.P.

Literature: (Paul et al. 1985a)

Pseudocercospora withaniae (Syd. \& P. Syd.) Deighton, Mycol. Pap. 140: 155 (1976)

= Cercospora withaniae Syd. \& P. Syd., Annls mycol. 10(5): 444 (1912)

Host: On leaves of Withania somnifera (L.) Dunal. (Solanaceae)

Location: Solan (Solan) H.P.

Literature: (Paul \& Bhardwaj 1986b)

Ramularia Unger, Exanth. Pflanzen (Wien): 119 (1833)

Fig. 7

\section{Description \& Illustration}

Ramularia is a genus of hyphomycetes fungi that harbours plant pathogens responsible for yield losses to many important crops. Disease symptoms of these fungi appear as small brown spots, surrounded by a yellow halo on the leaf surface. Later, neighbouring spots join together to form large brown patches. These fungi produce hyaline conidiophores and conidia with distinct, thick, dark, refractive conidiogenous loci and conidial hila. Mycosphaerella is sexual morph of these fungi. Microscopic characteristics include internal (external also) mycelium, superficial branched, septate, hyaline, smooth hyphae; stromata almost lacking to well-developed; Conidiophores in small to moderately large fascicles, loose to moderately dense, erect, straight, subcylindrical to geniculate-sinuous, unbranched or occasionally branched, hyaline, thin-walled, smooth, arising from internal hyphae or stromata, emerging through stomata; Conidia solitary or catenate, short conidia narrowly ellipsoid-ovoid to subcylindrical, longer conidia cylindrical, hyaline, thin-walled, verruculose, apex obtuse, rounded to truncate, base obconically truncate, slightly thickened and darkened hila. Total 1179 epithets are available with Index fungorum for this genus (Index Fungorum 2019 accessed on 06.12.2019).

Type species: Ramularia pusilla Unger, Exanth. Pflanzen (Wien): 169 (1833)

Ramularia decipiens Ellis \& Everh., J. Mycol. 1(5): 70 (1885)

Host: Leaves of Rumex orientalis Bernh. ex Schult. \& Schult.f. \& $R$. nepalensis Spreng. (Polygonaceae); Ranunculus laetus Wall. ex Hook. f. \& J.W. Thomson (Ranunculaceae)

Location: Shimla (Shimla) and Solan (Solan), H.P.

Literature: (Padwick 1946, Paul \& Sharma 1999)

Ramularia didyma Unger, Exanth. Pflanzen (Wien): 169 (1833)

= Didymaria didyma (Unger) Pound, Am. Nat. 23: 163 (1889)

= Ramularia didyma f. ranunculi-repentis Thüm., Mycoth. Univ., cent. 30: no. 2976 (1881)

= Ramularia ranunculi Peck, Ann. Rep. N.Y. St. Mus. nat. Hist. 35: 141 (1884)

= Ramularia calthae Lindr., Acta Soc. Fauna Flora fenn. 23(no. 3): 15 (1902)

= Ramularia didyma var. pulsatillae (Hollós) U. Braun, Monogr. Cercosporella, Ramularia

Allied Genera (Phytopath. Hyphom.) 2: 240 (1998)

= Ramularia exigua U. Braun, Mycotaxon 51: 57 (1994)

= Ramularia didyma var. exigua (U. Braun) U. Braun, Monogr. Cercosporella, Ramularia

Allied Genera (Phytopath. Hyphom.) 2: 240 (1998) 
Host: Leaves of Ranunculus leatus Wall. ex. Hook. f. \& J.W. Thomson (Ranunculaceae)

Location: Solan (Solan), H.P.

Literature: (Munjal \& Sharma 1976)

Ramularia gossypii (Speg.) Cif., Atti Ist. bot. Univ. Lab. crittog. Pavia, sér. 5 19: 124 (1962)

= Septocylindrium gossypii (Speg.) Subram., Hyphomycetes (New Delhi): 309 (1971)

= Ramulariopsis gossypii (Speg.) U. Braun, Nova Hedwigia 56(3-4): 432 (1993)

= Septocylindrium areola (G.F. Atk.) Pound \& Clem., Minn. bot. Stud. 1(Bulletin 9): 651 (1896)

= Symphyosira areola (G.F. Atk.) Sawada, Special Publication College of Agriculture, National Taiwan University 8: 232 (1959)

= Mycosphaerella areola Ehrlich \& F.A. Wolf, Phytopathology 22: 238 (1932)

Host: Leaves of Gossypium sp. (Malvaceae)

Location: Bilaspur (Bilaspur), H.P.

Literature: (Paul et al. 1985c)

Ramularia lineola Peck, Ann. Rep. N.Y. St. Mus. nat. Hist. 32: 43 (1880) [1879]

Host: Leaves of Taraxacum officinale (L.) Wigg. (Asteraceae)

Location: Solan (Solan), H.P.

Literature: (Munjal \& Sharma 1976)

Ramularia narkandensis Deighton, Trans. Br. mycol. Soc. 60(1): 162 (1973)

Host: Leaves of Fragaria vesca L. (Rosaceae);

Location: Narkanda (Shimla), H.P.

Literature: (Deighton 1973)

Ramularia phaseoli (O.A. Drumm.) Deighton, Trans. Br. mycol. Soc. 50(1): 125 (1967)

Host: Leaves of Phaseolus vulgaris L. (Fabaceae); Phaseolus mungo (L.) Hepper (Fabaceae)

Location: Solan (Solan), H.P.

Literature: (Sohi et al. 1965, Paul \& Bhardwaj 1986a)

Ramularia rufomaculans Peck, Ann. Rep. N.Y. St. Mus. nat. Hist. 34: 46 (1883) [1881]

= Ramularia rufomaculans var. gallica Sacc., G. bot. ital., n.s. 23(2): 229 (1916)

= Ramularia rufomaculans var. longispora U. Braun \& C.F. Hill, Mycol. Progr. 1(1): 26 (2002)

= Septocylindrium rufomaculans (Peck) Pound \& Clem., Minn. bot. Stud. 1 (Bulletin 9): 651 (1896)

Host: Leaves of Polygonum pterocarpum Wall. (Polygonaceae)

Location: Solan (Solan), H.P.

Literature: (Paul \& Sharma 1999)

Ramularia variabilis Fuckel, Jb. nassau. Ver. Naturk. 23-24: 361 (1870) [1869-70]

= Cylindrosporium variabilis (Fuckel) J. Schröt. [as 'Cylindrospora'], in Cohn, Krypt.-Fl.

Schlesien (Breslau) 3.2(4): 490 (1897) [1908]

= Entylomella variabilis (Fuckel) Cif., Annls mycol. 26(1/2): 17 (1928)

= Ovularia variabilis (Fuckel) E. Bommer \& M. Rousseau, Flora mycol. Bruxelles: 274

(1884)

Host: Leaves of Verbascum thapsus L. (Scrophulariaceae)

Location: Solan (Solan), H.P.

Literature: (Munjal \& Sharma 1976, Paul \& Sharma 1999) 

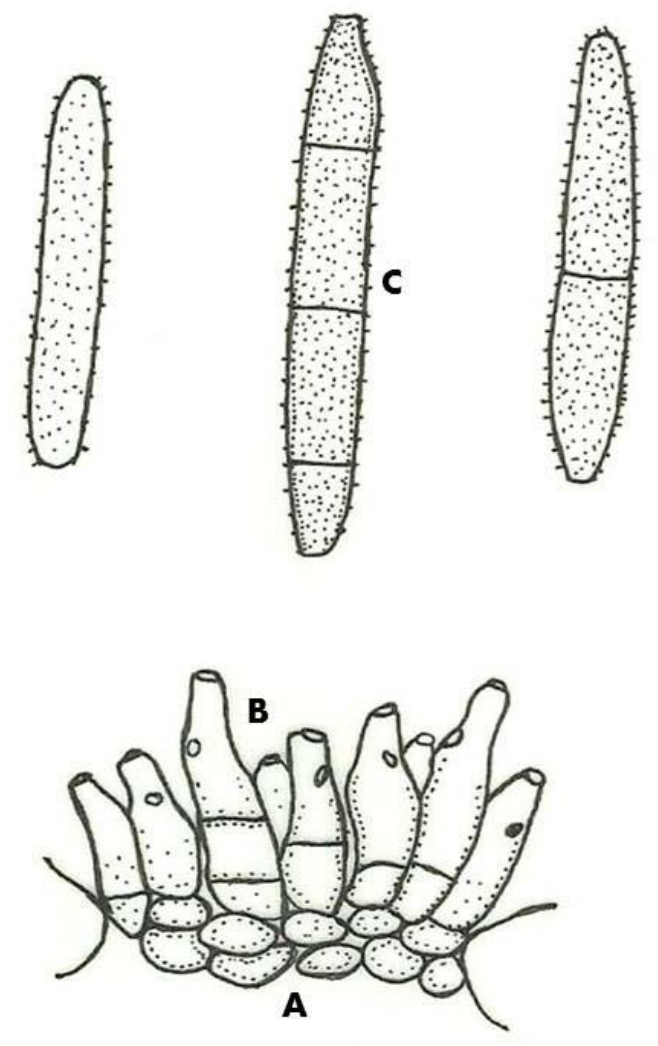

Fig. 7 - Ramularia didyma. A Conidiophore fascicles. B Conidiophore tips. C Conidia. (Braun 1998)

Rosisphaerella Videira \& Crous, Studies in Mycology, 87: 257-421 (2017)

\section{Description \& Illustration}

Rosisphaerella is assembly of phytopathogenic, foliicolous fungi reported to cause diseases on plant leaves as circular or irregular leaf spots. These fungi possess intyernal mycelium composed of hyaline and pale brown to brown, septate, branched hyphae. Stromata absent or small, epidermal, substomatal, brown to dark brown, if present. Conidiophores arises as solitary to fascicule, often synnematous, dark olivaceous brown near base and paler toward the tip, smooth, simple, multiseptate, straight to sinuous, usually geniculate-sinuous from stromata or few brown cells. Conidiogenous cells bears single pale to medium olivaceous brown, smooth to finely verruculose, cylindrical to obclavate, straight to mildly curved, septate conidia, obconically truncate at base and rounded at apex, hila somewhat thickened and darkened (Videira et al. 2017). As this is newly reported genus of cercosporid fungi, onle one record of type speies namely, Rosisphaerella rosicola is available (Index Fungorum 2019, accessed on 06.12.2019).

Type species: Rosisphaerella rosicola (Pass.) U. Braun, C. Nakash., Videira \& Crous 2017

Rosisphaerella rosicola (Pass.) U. Braun, C. Nakash., Videira \& Crous, in Videira, Groenewald, Nakashima, Braun, Barreto, de Wit \& Crous, Stud. Mycol. 87: 350 (2017)

= Cercospora rosicola Pass. [as 'rosaecola'], in Thümen, Just's Bot. Jahresber. 3: 276 (1877)

= Mycosphaerella rosicola (Pass.) B.H. Davis, Mycologia 30(3): 296 (1938)

= Phaeosphaerella rosicola (Pass.) Tomilin, Opredelitel’ Gribov roda Mycosphaerella Johans: 285 (1979)

= Phaeosporella rosicola (Pass.) Tomilin, Opredelitel' Gribov roda Mycosphaerella Johans: 285 (1979)

= Passalora rosicola (Pass.) U. Braun, Mycotaxon 55: 234 (1995) 
Host: On leaves of Rosa indica L. (Rosaceae); Smilax aspera L. (Smilacaceae).

Location: Mandi (Mandi) H.P.

Literature: (Chona et al. 1959, Singh et al. 1999)

Sirosporium Bubák \& Serebrian., Hedwigia 52: 273 (1912) total 34 epithets on 15.3.19

Fig. 8

\section{Description \& Illustration}

Sirosporium is a plant pathogenic fungi produces disease symptoms on leaves as effuse, velvety, oilvaceous, radish or dark blackish brown spots or colonies. Mycelium partially submerged or superficial with macronematous, micronematous or mononematous, branched or unbranched, plae to mid brown, smooth or verrucose conidiophores. Conidia simple, solitary, dry, cylindrical with rounded ends, septate transversely or sometime longitudinally, smooth, ruguse or verrucose. There are about 34 epithets reported worldwide in the genus Sirosporium (Index Fungorum 2019 accessed on 06.12.2019).

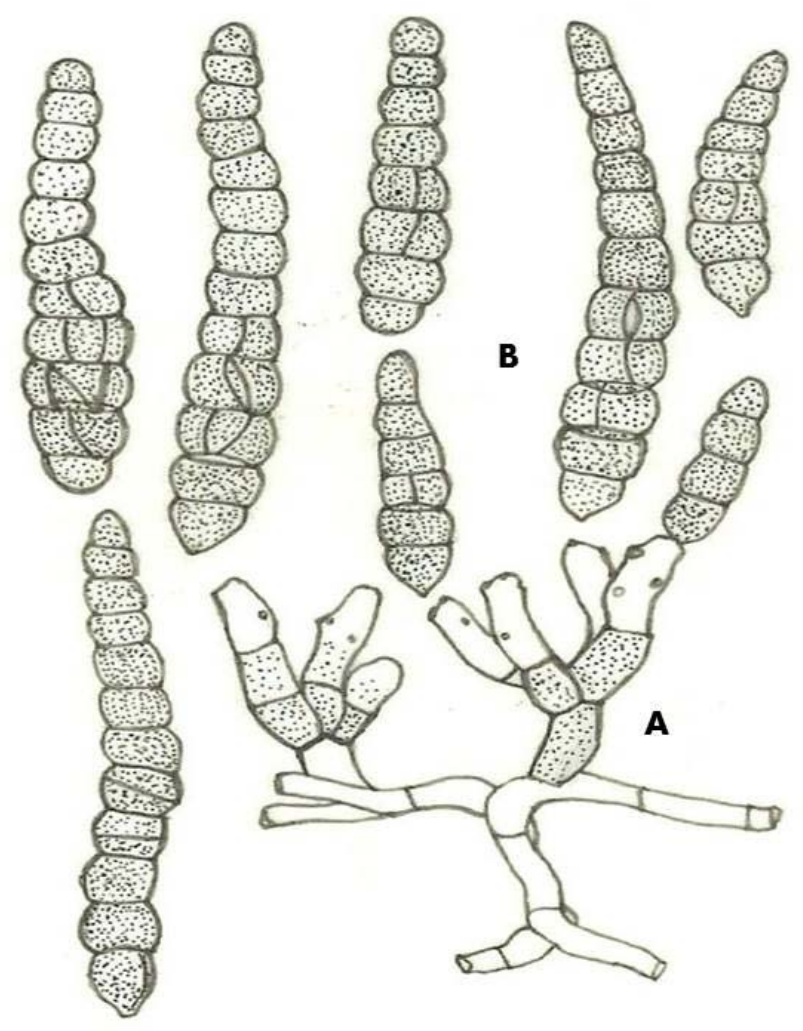

Fig. 8 - Sirosporium antenniformae. A Conidiophores. B Conidia. (Seifert et al. 2011)

Type species: Sirosporium antenniformae (Berk. \& Curt.) Bubak \& Serebrianikow, in M.B. Ellis Mycol. Pap., 87: 2-11. 1963.

Sirosporium celtidis (Biv.) M.B. Ellis, Mycol. Pap. 87: 4 (1963)

Host: Leaves Celtis occidentalis L. (Cannabaceae).

Location: Shimla (Shimla), H.P.

Literature: (Paul et al. 1986, Kamal 2010)

Sirosporium mori (Syd. \& P. Syd.) M.B. Ellis, Mycol. Pap. 87: 7 (1963)

Host: Leaves of Morus alba L. (Moraceae).

Location: Chauntra (Mandi), H.P.

Literature: (Paul \& Singh 1986, Paul et al. 1986, Kamal 2010) 


\section{Description \& Illustration}

Stigmina is a foliicolous genus of plant pathogenic fungi. These fungi appear underside of leaves as punctiform, brown to black colonies. Mycelium mostly internal, septate, hyaline to brown, immersed in or beneath stromata. Stromata well developed, emerged, erumpent or superficial, subhyaline to dark brown. Condiophores absent or macronematous, short and packed closely to form sporodochia, smooth or verrucose, unbranched, straight or flexious, hyaline to brown or olivaceous brown. Conidia solitary, apical, dry or formed in gelatinous matrix, acrogenous, simple, cylindrical, brown, rounded at apex, truncate at the base, smooth to verruculose, transversely septate and sometimes 1 or more oblique septation. There are 165 epithets reported worldwide in the genus Stigmina (Index Fungorum 2019 accessed on 06.12.2019).

Type species: Stigmina platani (Fuckel) Sacc., Michelia 2(no. 6): 22 (1880)

Stigmina ardisiae Munjal \& Kulshr., Phytopathology 21(3): 309 (1968)

Host: Leaves of Ardisia solanacea Roxb. (Primulaceae)

Location: Kangra (Kangra), H.P.

Literature: (Munjal \& Kulshreshtha 1968)

Stigmina carpophilla (Lév.) M.B. Ellis, Mycol. Pap. 72: 56 (1959)

Host: Leaves of Prunus persica (L.) Batsch \& Prunus domestica L. (Rosaceae)

Location: Nauni (Solan), H.P.

Literature: (Sharma \& Paul 1986)

Stigmina terminaliae Munjal \& Kulshr., Phytopathology 21(3): 312 (1968)

Host: Leaves of Terminalia chebula Retz. (Combretaceae)

Location: Palampur (Kangra), H.P.

Literature: (Munjal \& Kulshreshtha 1968)

Teratosphaeria Syd. \& P. Syd., Annales Mycologici 10 (1): 39 (1912)

\section{Description \& Illustration}

Teratosphaeria is a genus of foliicolous fungi causing leaf spot dieases as subcircular to irregular, pale brown to almost gray spots on both surfaces of leaves. Stromata usually lacking on lower leaf surface and present as subglobular, brown on upper surface of leaves. Conidiophores subhyaline to very pale yellowish olivaceous, emerges solitary to fascicule, sparingly septate, curved to tortuous, unbranched to slightly branched. Conidia pale olivaceous to hyaline, cylindrical to attenuated, straight to curved with subtruncate to long obconically truncate base (Sydow \& Sydow 1912). Total 99 epithets are recorded wiroldwide for this genus (Index Fungorum 2019 accessed on 06.12.2019).

Type species: Teratosphaeria fibrillosa Syd. \& P. Syd. 1912

Teratosphaeria eucalypti (Cooke \& Massee) Crous, Persoonia 23: 115 (2009) (1989)

= Pseudocercospora eucalypti (Cooke \& Massee) Y.L. Guo \& X.J. Liu, Mycosystema 2: 234

= Kirramyces eucalypti (Cooke \& Massee) J. Walker, B. Sutton \& Pascoe, Mycol. Res. 96(11): 920 (1992)

= Phaeophleospora eucalypti (Cooke \& Massee) Crous, F.A. Ferreira \& B. Sutton, S. Afr. J. Bot. 63(3): 113 (1997)

= Stagonospora pulcherrima (Gadgil \& M.A. Dick) H.J. Swart, Trans. Br. mycol. Soc. 90(2): 
285 (1988)

$=$ Readeriella pulcherrima (Gadgil \& M.A. Dick) Crous \& U. Braun, in Crous, Braun \& Groenewald, Stud. Mycol. 58: 26 (2007)

Host: On leaves of Eucalyptus sp. (Myrtaceae).

Location: Solan (Solan) H.P.

Literature: (Paul \& Sharma 1999)

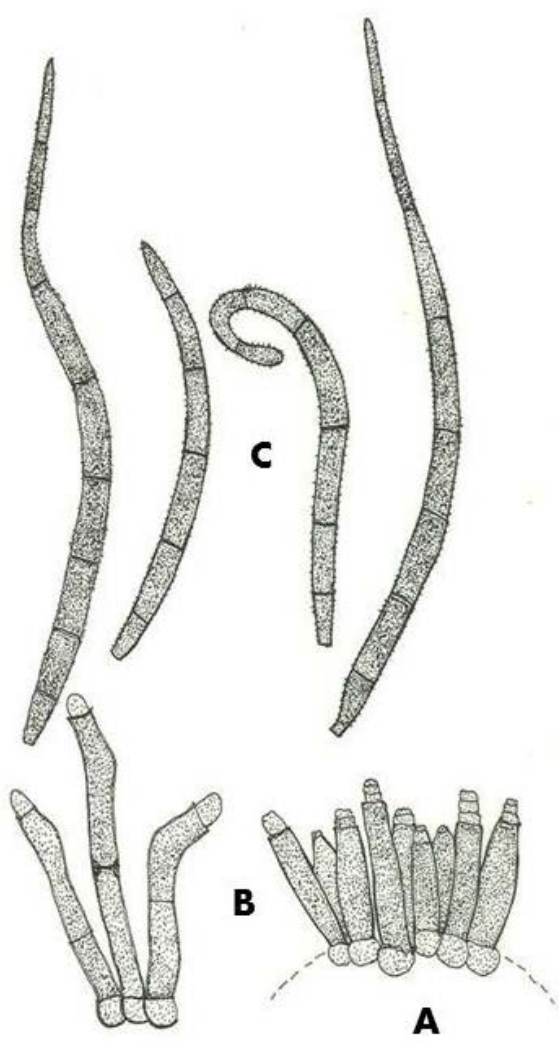

Fig. 9 - Stigmina curvispora. A Conidiophore fascicles. B Conidiophores. C Conidia. (Braun et al 2016)

\section{Host and Fungus Index}

In this section scientific name of host plants along with the cercosporoid fungi occurring on them are provided in table 2 .

Table 2 Host and fungus index for cercosporoid fungi occurring in Himachal Pradesh

\begin{tabular}{cll}
\hline Sr. No. & Host Plant & Cercosporoid fungi \\
\hline 1. & Abelmoschus esculentus & Pseudocercospora abelmoschi (Ell. \& Everh.) Dieghton \\
2. & Achyranthes aspera & Cercospora achyranthina Thirum. \& Chupp. \\
3. & Adhatoda vasica & Cercospora adhatodae S. Chowdhury \\
4. & Ageratina adenophora & $\begin{array}{l}\text { Pseudocercospora eupatoriicola (Govindu \& Thirum.) A.Z.M. } \\
\text { Khan \& Shamsi }\end{array}$ \\
5. & Albizia lebbek & Cercospora albiziae A.K. Kar \& M. Mandal \\
6. & Allium tuberosum & Cercospora duddiae Welles \\
7. & Althea rosea & Cercospora nebulosi Sacc. \\
8. & Amaranthus sp. & Cercospora brachiate Ellis \& Everh. \\
9. & Arachis hypogaea & Cercospora arachidicola Hori; Nothopassalora personata (Berk. \\
& & \& M.A. Curtis) U. Braun \\
\hline
\end{tabular}


Table 2 Continued.

\begin{tabular}{|c|c|c|}
\hline Sr. No. & Host Plant & Cercosporoid fungi \\
\hline 10. & Ardisia solanacea & Stigmina ardisiae Munjal \& Kulshr. \\
\hline 11. & Asparagus officinalis & Cercospora asparagi Sacc. \\
\hline 12. & Azadirachta indica & $\begin{array}{l}\text { Cercospora leucosticta Ellis \& Everh.; Pseudocercospora } \\
\text { subsessilis (Syd. \& P. Syd.) Deighton }\end{array}$ \\
\hline 13. & Beta cicla & Mycovellosiella bellynckii (Westend.) Constant. \\
\hline 14. & Beta vulgais L. var. mangel & Cercospora beticola Sacc \\
\hline 15. & Brassica rapa & $\begin{array}{l}\text { Neopseudocercosporella capsellae (Ellis \& Everh.) Videira \& } \\
\text { Crous }\end{array}$ \\
\hline 16. & Buddleia sp. & $\begin{array}{l}\text { Pseudocercospora blumeae (Thüm.) Deighton; } \\
\text { Pseudocercospora buddlejae (W. Yamam.) Goh \& W.H. Hsieh }\end{array}$ \\
\hline 17. & Cannabis sativa & Pseudocercospora cannabina (Wakef.) Deighton \\
\hline 18. & Capsicum annuum & Cercospora capsici Heald \& F.A. Wolf \\
\hline 19. & Cassia tora & Passalora occidentalis (Cooke) U. Braun \\
\hline 20. & Celtis occidentalis & Sirosporium celtidis (Biv.) M.B. Ellis \\
\hline 21. & Clemaits gouriana & Passalora squalidula (Peck) U. Braun \\
\hline 22. & Cucumis sativus & Cercospora citrullina Cooke \\
\hline 23. & Cucurbita moschata & Cercospora citrullina Cooke \\
\hline 24. & Datura metel & Cercospora daturicola (Speg.) Vassiljevsky \\
\hline 25. & Datura stramonium & Cercospora daturicola (Speg.) Vassiljevsky \\
\hline 26. & Dianthus caryophyllus & Pseudocercospora diclipterae (A.K. Kar \& M. Mandal) Deighton \\
\hline 27. & Dicliptera roxburghiana & Cercospora dianthi A.S. Mull. \& Chupp \\
\hline 28. & Digitalis purpurea & Cercospora digitalis P.K. Chi \& C.K. Pai \\
\hline 29. & Dodonaea viscosa & Cercospora mitteriana Syd. \\
\hline 30. & Dolichos biflorus & $\begin{array}{l}\text { Cercospora kikuchii (Tak. Matsumoto \& Tomoy.) M.W. } \\
\text { Gardner; Mycosphaerella cruenta Latham }\end{array}$ \\
\hline 31. & Eucalyptus sp. & Teratosphaeria eucalypti (Cooke \& Massee) Crous \\
\hline 32. & Flacourtia indica & Cercospora doryalidis Chupp \& Doidge \\
\hline 33. & Fragaria vesca & Ramularia narkandensis Deighton \\
\hline 34. & Geranium sp. & $\begin{array}{l}\text { Cercospora geranii Kellerm. \& Swingle; Pseudocercospora } \\
\text { geraniicola U. Braun }\end{array}$ \\
\hline 35. & Gerbera sp. & Cercospora gerberae Chupp \& Viégas \\
\hline 36. & Glycine max & Cercospora kikuchii (Tak. Matsumoto \& Tomoy.) M.W. Gardner \\
\hline 37. & Gossypium sp. & Ramularia gossypii (Speg.) Cif. \\
\hline 38. & Helianthus annuus & Cercospora helianthicola Chupp \& Viégas \\
\hline 39. & Hibiscus rosa- sinensis & Pseudocercospora abelmoschi (Ell. \& Everh.) Dieghton \\
\hline 40. & Hydrangea sp. & Cercospora hydrangeae Ellis \& Everh. \\
\hline 41. & Impatiens balsamina & Cercospora fukushiana (Matsuura) W. Yamam. \\
\hline 42. & Impatiens gigantea & Cercospora fukushiana (Matsuura) W. Yamam. \\
\hline 43. & Justicia sp. & Cercospora justiciicola F.L. Tai \\
\hline 44. & Lathyrus aphaca & $\begin{array}{l}\text { Passalora lathyri-aphacae (Lall, H.S. Gill \& Munjal) U. Braun } \\
\text { \& Crous }\end{array}$ \\
\hline 45. & Lygodium japonicum & Cercospora lygodiicola Lall, J.N. Kapoor \& Munjal \\
\hline 46. & Melothria heterophylla & Cercospora melothriae Sawada \\
\hline 47. & Momordica charantia & Cercospora citrullina Cooke \\
\hline 48. & Morus alba & $\begin{array}{l}\text { Pseudocercospora mori (Hara) Deighton; Sirosporium } \\
\text { mori (Syd. \& P. Syd.) M.B. Ellis }\end{array}$ \\
\hline 49. & Murraya koenigii & Pseudocercospora murrayae (A.K. Kar \& M. Mandal) Deighton \\
\hline 50. & Ocimum tenuiflorum & Pseudocercospora ocimicola (Petr. \& Cif.) Deighton \\
\hline 51. & Oplismenus sp. & Cercospora oplismeni Lall, H.S. Gill \& Munjal \\
\hline 52. & Phaseolus mungo & Ramularia phaseoli Klotzsch \\
\hline 53. & Phaseolus vulgaris & Ramularia phaseoli Klotzsch \\
\hline 54. & Physalis minima & Cercospora physalidicola Ellis \& Barthol. \\
\hline 55. & Pistacia integrrima & Cercospora megaspermae Bhardwaj \& Sharma \\
\hline 56. & Pisum sativum & Cercospora pisi-sativi J.A. Stev. \\
\hline
\end{tabular}


Table 2 Continued.

\begin{tabular}{|c|c|c|}
\hline Sr. No. & Host Plant & Cercosporoid fungi \\
\hline 57. & Platanus orientalis & Pseudocercospora platanigena Videira \& Crous \\
\hline 58. & Plumeria tomentosa & Pseudocercospora plumeriae (Chupp) Tak. Kobay. \\
\hline 59. & Polygonum aviculare & Passalora avicularis (G. Winter) Crous \\
\hline 60. & Polygonum pterocarpum & Ramularia rufomaculans Peck. \\
\hline 61. & Polygonum sp. & Pseudocercospora paludicola (Speg.) U. Braun \\
\hline 62. & Populus yunnanensis & Mycosphaerella cruenta Latham \\
\hline 63. & Prunus armeniaca & $\begin{array}{l}\text { Mycosphaerella pruni-persicae Deighton; Cercosporella } \\
\text { persicae (Sacc.) Sacc. }\end{array}$ \\
\hline 64. & Prunus domestica & Stigmina carpophilla (Lév.) M.B. Ellis \\
\hline 65. & Prunus persica & $\begin{array}{l}\text { Cercospora persica Sharma \& Paul; Stigmina carpophilla (Lév.) } \\
\text { M.B. Ellis; Mycosphaerella cerasella Aderh. }\end{array}$ \\
\hline 66. & Punica granatum & Pseudocercospora punicae (Henn.) Deighton \\
\hline 67. & Ranunculus laetus & Ramularia decipiens Ellis \& Everh.; Ramularia didyma Unger \\
\hline 68. & Ricinus communis & Cercospora ricinella Sacc. \& Berl. \\
\hline 69. & Rosa indica & Rosisphaerella rosicola (Pass.) U. Braun \\
\hline 70. & Rumex nepalensis & Cercospora citrullina Cooke; Ramularia decipiens Ellis \& Everh. \\
\hline 71. & Rumex orientalis & Cercospora citrullina Cooke; Ramularia decipiens Ellis \& Everh. \\
\hline 72. & Salix tetrasperma & Pseudocercospora salicina (Ellis \& Everh.) Deighton \\
\hline 73. & Salmalia malabarica & Cercospora bombacicola Munjal, Lall \& Chona \\
\hline 74. & Sesamum indicum & Cercospora sesami Zimm. \\
\hline 75. & Smilax aspera & $\begin{array}{l}\text { Cercospora physalidis Ellis; Passalora pallidissima (Chupp) U. } \\
\text { Braun; Rosisphaerella rosicola (Pass.) U. Braun; } \\
\text { Neocercosporidium smilacis (Thüm.) U. Braun, C. Nakash., } \\
\text { Videira \& Crous }\end{array}$ \\
\hline 76. & Smilax sp. & $\begin{array}{l}\text { Pseudocercospora mississippiensis (Tracy \& Earle) R.F. } \\
\text { Castañeda \& U. Braun }\end{array}$ \\
\hline 77. & Solanum melongena & Cercospora melongenae Welles \\
\hline 78. & Solanum nigrum & $\begin{array}{l}\text { Cercosporia nirgi var. microsporae Bhardwaj \& Paul; } \\
\text { Cercosporia nirgi var. microspora Bhardwaj \& Paul; Cercospora } \\
\text { solani Thüm. }\end{array}$ \\
\hline 79. & Solanum tuberosum & $\begin{array}{l}\text { Cercospora solani-tuberosi Thirum.; Cercospora solanicola G.F. } \\
\text { Atk.; Passalora concors (Casp.) U. Braun \& Crous; } \\
\text { Distomycovellosiella brachycarpa (Syd.) U. Braun, C. Nakash. }\end{array}$ \\
\hline 80. & Sonchus oleraceus & Cercospora sonchi Chupp \\
\hline 81. & Syzigium cumini & Cercospora eugeniae Chupp \\
\hline 82. & Tagetes patula & Cercospora tageticola Ellis \& Everh. \\
\hline 83. & Taraxacum officinale & Ramularia lineola Peck \\
\hline 84. & Terminalia chebula & Stigmina terminaliae Munjal \& Kulshr. \\
\hline 85. & Trigonella foenum-graecum & Cercospora traversoana Sacc. \\
\hline 86. & Verbascum thapsus & Ramularia variabilis Fuckel \\
\hline 87. & Verbena sp. & Passalora verbeniphila (Speg.) Crous \& U. Braun \\
\hline 88. & Veronica cinerea & Pseudocercospora cinerea (Pavgi \& U.P. Singh) Deighton \\
\hline 89. & Vigna sinensis & Cercospora canescens Ellis \& G. Martin \\
\hline 90 . & Vigna vexillata & Cercospora canescens Ellis \& G. Martin \\
\hline 91. & Vigna unguiculata & Pseudocercospora dolichi (Ellis \& Everh.) J.M. Yen \\
\hline 92. & Viola serpens & Cercospora violae Sacc. \\
\hline 93. & Vitis vinifera & Pseudocercospora riachueli (Speg.) Deighton \\
\hline 94. & Withania somnifera & Pseudocercospora withaniae (Syd. \& P. Syd.) Deighton \\
\hline 95. & Woodfordia floribunda & Pseudocercospora sydowiana (Chupp) U. Braun \& Crous \\
\hline 96. & Zanthoxylum alatum & Cercospora oxyphylli Pavgi \& U.P. Singh \\
\hline 97. & Zea mays & $\begin{array}{l}\text { Cercospora sorghi Ellis \& Everh.; Cercospora zeae- } \\
\text { maydis Tehon \& E.Y. Daniels }\end{array}$ \\
\hline 98. & Zinnia elegans & Cercospora zinniae Ellis \& G. Martin \\
\hline
\end{tabular}


Table 2 Continued.

\begin{tabular}{cll}
\hline Sr. No. & \multicolumn{1}{c}{ Host Plant } & \multicolumn{1}{c}{ Cercosporoid fungi } \\
\hline 99. & Zizyphus jujube & Pseudocercospora jujubae (S. Chowdhury) A.Z.M. Khan \& \\
& & Shamsi \\
100. & Zizyphus oenoplia & $\begin{array}{l}\text { Pseudocercospora jujubae (S. Chowdhury) A.Z.M. Khan \& } \\
\end{array}$
\end{tabular}

\section{Acknowledgements}

Authors are grateful to their respective organizations for providing the necessary laboratory facilities and valuable support during the study. We thank the anonymous reviewers for their critical comments and suggestions.

\section{References}

Agrios GN. 1997 - Plant Pathology 4th ed., Academic Press, San Diego, CA. 635 pp.

Ahanger RA, Bhat HA, Bhat TA, Ganie SA et al. 2013 - Impact of Climate Change on Plant Diseases. International Journal of Modern Plant \& Animal Sciences1, 105-115.

Bakhshi M, Arzanlou M, Babai-Ahari A. 2012 - Comprehensive check list of Cercosporoid fungi from Iran. Plant Pathology \& Quarantine 2, 44-55

Bakhshi M. 2019 - Epitypification of Cercospora rautensis, the causal agent of leaf spot disease on Securigera varia, and its first report from Iran. Fungal Systematics and Evolution 3, 157-163.

Bakshi BK, Reddy MAR, Singh B. 1970 - An unrecorded decay in livinp deodar. Indian Forester 96: 72-74.

Bhardwaj LN, Paul YS. 1986 - Pathological problems of Siris. (Albizzia lebbek Benth.) in Himachal Pradesh. In: National Symposium on Researches in Social Forestry for Rural Development. New Delhi, 1-2 January 1986, p. 18-19 (Abst.).

Bhardwaj LN, Paul YS. 1987 - Cercospora nigri Tharp Var. Microsporae Var. Nov. Causing Leaf Spot of Solanum nigrum L. Indian Forester 113, 509-510.

Bhardwaj LN, Sharma RC. 1994 - Some new fungal diseases of Pistacia integerrima from Himachal Pradesh. Indian Forester 120, 545-547.

Braun U. 1995 - A monograph of Cercosporella, Ramularia and allied genera (Phytopathogenic Hyphomycetes), Vol. 1. Eching bei Munchen: IHW Verlag.

Braun U. 1998 - A monograph of Cercosporella, Ramularia and allied genera (Phytopathogenic Hyphomycetes), Vol. 2. Eching bei Munchen: IHW Verlag.

Braun U, Crous PW, Nakashima C. 2016 - Cercosporoid fungi (Mycosphaerellaceae) 5. Species on dicots (Anacardiaceae to Annonaceae). IMA Fungus 7, 161-216.

Braun U, Crous PW. 2016 - (2415) Proposal to conserve the name Cercospora (Ascomycota: Mycosphaerellaceae) with a conserved type. Taxon 65(1), 185.

Braun U, Nakashima C, Crous PW. 2013 - Cercosporoid fungi (Mycosphaerellaceae) 1. Species on other fungi, Pteridophyta and Gymnospermae. IMA Fungus 4, 265-345.

Chakraborty S, Murray GM, Magarey PA, Yonow T et al. 1998 - Potential impact of climate change on plant diseases of economic significance to Australia. Australian Plant Pathology 27, 15-35.

Chandel SS. 1998 - Hitherto unrecorded diseases of carnation from Himachal Pradesh. Indian Journal of Plant Pathology 16, 70-71.

Chona BL, Lall G, Munjal RL. 1959 - Some Cercospora species from lndia-1. Indian Phytopathology 12, 76-84.

Coakley SM, Scherm H, Chakraborty S. 1999 - Climate change and plant disease management. Annual Review of Phytopathology 37, 399-426

Crous PW, Aptroot A, Kang J, Braun U, Wingfield MJ. 2000 - The genus Mycosphaerella and its anamorphs. SllIdies in Mycology 45, 107-121. 
Crous PW, Braun U. 2003 - Mycosphaerella and its Anamorphs, 1. Names published in Cercospora and Passalora. CBS Biodiversity Series 1. Utrecht, Netherland.

Deighton PC. 1973 - Ramularia narkandensis sp. nov. on Fragaria in India. Transactions of British Mycological Society 60, 162-164.

Dhancholia S, Singh S. 1992 - Outbreak of Cercospora abelmoschi Ell \& Ev. in Himachal Pradesh. Himachal Journal of Agricultural Research 18, 60.

Fries EM. 1849. Summa vegetabilium Scandinaviae. Sectio Posterior. Stockholm: A. Bonnier

Gangopadhyay S, Kapoor KS. 1976 - A new leaf spot disease of Brassica rapa in Kullu valley. Science \& Culture 42, 619-620.

Inácio CA, Dianese JC. 1999 - A new Mycovellosiella species on Myracrodruon urunduva. Mycotaxon 72, 253-263.

Kamal. 2010 - Cercosporoid fungi of India. Bishen Singh Mahendra Pal Singh, Dehradun, India.

Lall G, Gill HS, Munjal RL. 1961 - Some Cercospora spp. from India-V. Indian Phytopathology $14,115-119$.

Lall G, Gill HS, Munjal RL. 1962 - Some Cercospora spp. from India-VIII. Indian Phytopathol.15:243-245.

Lall G, Kapoor JN, Munjal RL. 1963 - A new leaf spot disease of maize in India. Indian Phytopathology 16, 240-241.

Lall G, Kapoor JN, Munjal RL.1964 - Some fern inhabiting Cercospora spp. from India. Indian Phytopathology 17, 181-183.

Munjal RL, Kulshreshtha DD. 1968 - Some dematiaceous hyphomycetes from India-V. Stigmins sp. Indian Phytopathology 21, 309-314.

Munjal RL, Seth KK. 1966 - Cercospora sp. causing leaf spot of grapes in India. Indian Phytopathology 19, 209-213.

Munjal RL, Sharma AD. 1976 - Additions to mycoflora of Himachal Pradesh-I. Himachal Journal of Agricultural Research 4, 87-91.

Munjal RL, Lall G, Chona BL. 1960 - Some Cercospora species from India-IV. Indian Phytopathology 13, 144-149.

Munjal RL, Sharma AD. 1975 - Mycoflora of conifer seeds. Indian Journal of Mycololgy and Plant Pathology 5, 145-148.

Padwick GW. 1946 - Notes on Indian Fungi-III. Mycological Papers. Imperial Mycological Institute 17, 12.

Paul YS, Bhardwaj LN. 1986a - Cucurbitaria berberidis on Berberis lycium. Indian Phytopathology 39, 320.

Paul YS, Bhardwaj LN. 1986b - Additions to fungi of Himachal Pradesh III. Himachal Journal of Agricultural Research 12, 150-153.

Paul YS, Sharma RC. 1999 - Commonly occurring fungi of Himachal Pradesh without mycological records. Dr. YS Pamnar Univ. of Hort. \& Fty. Solan. 10 pp.

Paul YS, Singh BM. 1986 - Additions to fungi of India. Indian Phytopathology 39(2): 317.

Paul YS, Bhardwaj LN, Sharma RC. 1990 - Additions to fungi of Himachal Pradesh - III (Hyphomycetes). Indian Journal of Plant Pathology 20, 196-199.

Paul YS. 1989 - Seed borne mycoflora of soybean and its control in Himachal Pradesh. Indian Journal of Mycology and Plant Pathology 192, 253-257.

Paul YS, Sugha SK, Pal J. 1985a - Fungi new to Himachal Pradesh - II. Mycologicai Herbarium specimens, Deptt. of Mycol. \& Plant Pathol. Dr. YSP UHF, Solan 3 pp.

Paul YS, Aganvala RK, Sharma RC. 1985b - Additions to fungi of Himachal Pradesh Hypomycetes I. Mycological Herbarium specimens, Deptt. Mycol. \& Plant Pathol. Dr. YSP UHF. Solan, 4 pp.

Paul YS, Aganvala RK, Sharma RC. 1985c - Additions to fungi of Himachal Pradesh Hypomycetes II. Mycological Herbarium specimens, Deptt. Mycol. \& Plant Pathol. Dr. YSP UHF. Solan, 4 pp. 
Paul YS, Sharnia RC, Bhardwaj LN. 1986 - Additions to fungi of Himachal Pradesh Hyphomycetes 111 Mycological Herbarium specimens. Deptt. of Mycol^ \& Plant Pathol. Dr. YSP UHF. Solan, 3 pp.

Phengsintham P, Chukeatirote E, McKenzie EHC, Hyde KD, Braun U. 2013 - Monograph of Cercosporoid fungi from Laos. Current Research in Environmental \& Applied Mycology 3, 34-158.

Philip T, Govindaiah, Saramangala HS. 1994b - Two new host records in mulberry. Indian Phytopathology 47, 214.

Philip T, Janardhan L, Govindaiah, Bajaj Ak. 1994a - Black leaf spot disease of mulberry- first report. Indian Journal of Sericulture 33, 186-187.

Prakasam V. 1991 - Red leaf spot of cinnamon in lower pulney hills of Tamil Nadu. Indian Cocoa, Aracenut, and Spices Journal 14, 123.

Rai RP. 1983 - Cercospora solanicola as a cause of tuber rot of potato in storage. Indian Phytopathology 36, 390-392.

Roy AK. 1965 - Additions to fungal flora of Assam-I. Indian Phytopathology 18, 327-334.

Saharan GS. 1979 - Seed borne mycoflora of horse gram (Dolichos biflorus L.). Indian Phytopathology 32, 138-140.

Seifert K, Morgan-Jones G, Gams W, Kendrick B. 2011 - The Genera of Hyphomycetes. CBSKNAW Fungal Biodiversity Centre, The Netherlands.

Sharma RC, Paul YS. 1986 - New diseases of peach (Prunus percica L.) from India. Indian Journal of Plant Patholology 4, 182.

Sharma AD. 1977 - Two new diseases of Dolichos biflorus in India. Indian Journal of Plant Patholology 7, 170.

Singh A. Paul YS, Dhancholia S. 1999 - Additions to fungi of Himachal Pradesh. National Symposium on Plant Disease Scenaria under changing Agroecosystems. HPKV Palampur, October 29-30, 1999.

Sivanesan A. 1984 - The Bitunicate Ascomycetes and their anamorphs. Lubrecht \& Cramer Ltd., Liechtenstein, pp182

Sohi HS, Gupta GK. 1966 - Additions to the fungi of Himachal Pradesh. Indian Phytopathology 19, $230-231$.

Sohi HS, Jain SS, Sharma SL, Verma BR. 1964 - New records of plant disease from Himachal Pradesh. Indian Phytopathology 17, 42-45.

Sohi HS, Sharma SL, Verma BR, Sachdev KB. 1965 - Leaf spot of bean caused by Ramularia phaseolus Petrak. IndianPhytopathology 18, 165.

Świderska-Burek U. 2015 - Cercosporoid fungi of Poland. Wrocław: Polish Botanical Society. Monographiae Botanicae 105, 1-164.

Sydow H, Butler EJ. 1907 - Fungi Indiane Orientalis Pars-I. Annals of Mycology 424-445

Sydow H, Mcrae W. 1929 - Hyphomycetes Indiae orientalis. Annales de Cryptogamie Exotique 2, 262-271.

Sydow H, Sydow P. 1912 - Beschreibungen neuer südafrikanischer Pilze. Annales Mycologici 10, 33-45.

Videira SIR, Groenewald JZ, Braun U, Shin HD, Crous PW. 2016 - All that glitters is not Ramularia. Studies in Mycology 83, 49-163.

Videira SIR, Groenewald JZ, Nakashima C, Braun U et al. 2017 - Mycosphaerellaceae - Chaos or clarity? Studies in Mycology 87, 257-421. 